\title{
One Dimensional Hyperbolic Systems of Conservation Laws
}

\author{
Alberto Bressan
}

\section{Introduction}

These notes are meant to provide a survey of some recent results and techniques in the theory of conservation laws. In one space dimension, a system of conservation laws can be written as

$$
u_{t}+f(u)_{x}=0 .
$$

Here $u=\left(u_{1}, \ldots, u_{n}\right)$ is the vector of conserved quantities while the components of $f=\left(f_{1}, \ldots, f_{n}\right)$ are called the fluxes. Integrating (1.1) over the interval $[a, b]$ one obtains

$$
\begin{aligned}
& \frac{d}{d t} \quad \int_{a}^{b} u(t, x) d x=\int_{a}^{b} u_{t}(t, x) d x=-\int_{a}^{b} f(u(t, x))_{x} d x \\
& \quad=f(u(t, a))-f(u(t, b))=[\text { inflow at } a]-[\text { outflow at } b] .
\end{aligned}
$$

In other words, each component of the vector $u$ represents a quantity which is neither created nor destroyed: its total amount inside any given interval $[a, b]$ can change only because of the flow across boundary points.

Systems of the form (1.1) can be used to express the fundamental balance laws of continuum physics, when small viscosity or dissipation effects are neglected [D2]. A primary example is provided by the Euler equations describing the evolution of a compressible, non viscous fluid:

$$
\left\{\begin{array}{lll}
\rho_{t}+(\rho v)_{x} & =0 & \text { (conservation of mass) } \\
(\rho v)_{t}+\left(\rho v^{2}+p\right)_{x} & =0 & \text { (conservation of momentum) } \\
(\rho E)_{t}+(\rho E v+p v)_{x} & =0 & \text { (conservation of energy) }
\end{array}\right.
$$

Here $\rho$ is the mass density, $v$ is the velocity while $E=e+v^{2} / 2$ is the energy density per unit mass. The system is closed by a constitutive relation of the form $p=p(\rho, e)$, determining the pressure as a function of the density and the internal energy. The particular values of $p$ depends on the gas under consideration.

Using the chain rule, (1.1) can be written in the quasilinear form

$$
u_{t}+A(u) u_{x}=0 \text {, }
$$


where $A(u) \doteq D f(u)$ is the Jacobian matrix of first order partial derivatives of $f$. For smooth solutions, the two equations (1.1) and (1.3) are entirely equivalent. However, if $u$ has a jump at a point $x_{0}$, the left hand side of (1.3) will contain the product of the discontinuous function $x \mapsto A(u(x))$ with the distributional derivative $u_{x}$, which in this case contains a Dirac mass at the point $x_{0}$. In general, such a product is not well defined. Hence (1.3) is meaningful only within a class of continuous functions. On the other hand, working with the equation in divergence form (1.1) allows us to consider discontinuous solutions as well, interpreted in distributional sense. More precisely, a locally integrable function $u=u(t, x)$ is a weak solution of (1.1) provided that

$$
\iint\left\{u \phi_{t}+f(u) \phi_{x}\right\} d x d t=0
$$

for every differentiable function with compact support $\phi \in \mathcal{C}_{c}^{1}$.

We say that the above system is strictly hyperbolic if every matrix $A(u)$ has $n$ real, distinct eigenvalues, say $\lambda_{1}(u)<\cdots<\lambda_{n}(u)$. In this case, one can find dual bases of left and right eigenvectors of $A(u)$, denoted by $l_{1}(u), \ldots, l_{n}(u)$ and $r_{1}(u), \ldots, r_{n}(u)$, normalized according to

$$
\left|r_{i}\right|=1, \quad l_{i} \cdot r_{j}= \begin{cases}1 & \text { if } i=j, \\ 0 & \text { if } i \neq j .\end{cases}
$$

To appreciate the effect of the non-linearity, consider first the case of a linear system with constant coefficients

$$
u_{t}+A u_{x}=0 .
$$

Call $\lambda_{1}<\cdots<\lambda_{n}$ the eigenvalues of the matrix $A$, and let $l_{i}, r_{i}$ be the corresponding left and right eigenvectors as in (1.5). One can then write the general solution of (1.6) as a superposition of independent linear waves:

$$
u(t, x)=\sum_{i} \phi_{i}\left(x-\lambda_{i} t\right) r_{i}, \quad \phi_{i}(y) \doteq l_{i} \cdot u(0, y) .
$$

Notice that here the solution is completely decoupled along the eigenspaces of $A$, and each component travels with constant speed, given by the corresponding eigenvalue of $A$.

In the nonlinear case (1.3) where the matrix $A$ depends on the state $u$, new features will appear in the solutions.

(i) Since the eigenvalues $\lambda_{i}$ now depend on $u$, the shape of the various components in the solution will vary in time (fig. 1). In particular, rarefaction waves will decay, and compression waves will become steeper, possibly leading to shock formation in finite time.

(ii) Since the eigenvectors $r_{i}$ also depend on $u$, nontrivial interactions between different waves will occur (fig. 2). In particular, the strength of the interacting waves may change, and new waves of different families can be created, as a result of the interaction. 


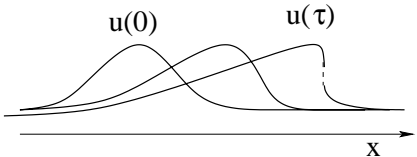

figure 1
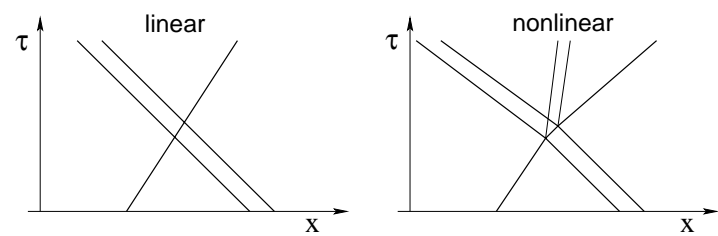

figure 2

Of all these effects, the most important for the mathematical analysis is the possible loss of regularity. This lack of regularity (also due to the absence of second order terms that could provide a smoothing effect), together with the strong nonlinearity of the equations, is the main source of the difficulties encountered in a rigorous mathematical analysis of this subject. Indeed, most of the powerful techniques of functional analysis do not apply in this context. Solutions cannot be represented as fixed points of continuous transformations, or in variational form, as critical points of suitable functionals. Dealing with vector valued functions, comparison principles based on upper or lower solutions cannot be used. Similarly, the theory of accretive operators and contractive nonlinear semigroups works well in the scalar case [C], but does not apply to systems. For the above reasons, the theory of hyperbolic conservation laws has largely developed by ad hoc methods, along two main lines.

1. The $B V$ setting, considered by Glimm [G]. Solutions are here constructed within a space of functions with bounded variation, controlling the $B V$ norm by a wave interaction functional.

2. The $\mathbf{L}^{\infty}$ setting, introduced by Tartar and DiPerna [DP2], based on weak convergence and a compensated compactness argument.

Both approaches yield results on the global existence of weak solutions. However, the method of compensated compactness appears to be suitable only for $2 \times 2$ systems. Moreover, it is only in the $B V$ setting that the well-posedness of the Cauchy problem could recently be proved, as well as the stability and convergence of vanishing viscosity approximations. In the remainder of this paper we thus concentrate on the theory of $B V$ solutions, referring to [DP2] or [Se] for the alternative approach based on compensated compactness.

Since the pioneering work of Glimm, the basic building block toward the construction and the analysis of more general solutions has been provided by the Riemann problem, i.e. the initial value problem with piecewise constant data

$$
u(0, x)=\bar{u}(x)=\left\{\begin{array}{lll}
u^{-} & \text {if } & x<0, \\
u^{+} & \text {if } \quad x>0 .
\end{array}\right.
$$

This was first introduced by B. Riemann (1860) in the context of isentropic gas dynamics. A century later, P. Lax [Lx] solved the Riemann problem for a general class of $n \times n$ systems. Solutions are self-similar, having the form

$$
u(t, x)=U(x / t) .
$$


The central position taken by the Riemann problem is related to a symmetry of the equations (1.1). If $u=u(t, x)$ is a solution of (1.1), then for any $\theta>0$ the function

$$
u^{\theta}(t, x) \doteq u(\theta t, \theta x)
$$

provides another solution. The solutions which are invariant under these rescalings of the independent variables are precisely those which correspond to some Riemann data.

Both the Glimm scheme and the method of front tracking yield approximate solutions of a general Cauchy problem by piecing together a large number of Riemann solutions. The approach is successful because one can provide an a priori bound on the total amount of new waves produced by nonlinear interactions, and hence on the total variation of the solution. It is safe to say that, in the context of weak solutions with small total variation, nearly all results on the existence, uniqueness, continuous dependence and qualitative behavior have relied on a careful analysis of the Riemann problem.

Very recently, in $[\mathrm{BB}]$ a substantially different perspective has emerged from the study of vanishing viscosity approximations. Solutions of (1.1) are sought as limits for $\varepsilon \rightarrow 0$ of solutions to the parabolic problems

$$
u_{t}^{\varepsilon}+A\left(u^{\varepsilon}\right) u_{x}^{\varepsilon}=\varepsilon u_{x x}^{\varepsilon} .
$$

Here $A(u) \doteq D f(u)$. This approach is very natural, and has been considered since the 1950's. However, it was only in the scalar case $[\mathrm{O}],[\mathrm{K}]$, that complete results could be obtained. For general $n \times n$ systems, the main difficulty lies in establishing the compactness of the approximating sequence. We observe that $u^{\varepsilon}(t, x)$ solves (1.8) if and only if $u^{\varepsilon}(t, x)=u(t / \varepsilon, x / \varepsilon)$ for some function $u$ which satisfies

$$
u_{t}+A(u) u_{x}=u_{x x} .
$$

In the analysis of vanishing viscosity approximations, the key step is to derive a priori estimates on the total variation and on the stability of solutions of (1.9). For this parabolic system, the rescaling $(t, x) \mapsto(\theta t, \theta x)$ no longer determines a symmetry. Hence the Riemann data no longer hold a privileged position. The role of basic building block is now taken by the viscous travelling profiles, i.e. the solutions of the form

$$
u(t, x)=U(x-\lambda t) .
$$

Of course, the function $U$ must then satisfy the second order O.D.E.

$$
U^{\prime \prime}=(A(U)-\lambda) U^{\prime} .
$$

The new point of view consists in decomposing the gradient $u_{x}$ locally as a sum of gradients of viscous travelling waves. More precisely, let a smooth function $u: \mathbb{R} \mapsto \mathbb{R}^{n}$ be given. At each point $x$, looking at the third order jet $\left(u, u_{x}, u_{x x}\right)$ we seek travelling profiles $U_{1}, \ldots, U_{n}$ such that

$$
U_{i}^{\prime \prime}=\left(A\left(U_{i}\right)-\sigma_{i}\right) U_{i}^{\prime}
$$

for some speed $\sigma_{i}$ close to the characteristic speed $\lambda_{i}$, and moreover

$$
U_{i}(x)=u(x) \quad i=1, \ldots, n,
$$




$$
\sum_{i} U_{i}^{\prime}(x)=u_{x}(x), \quad \sum_{i} U_{i}^{\prime \prime}(x)=u_{x x}(x) .
$$

It turns out that this decomposition is unique provided that the travelling profiles are chosen within suitable center manifolds. We let $\tilde{r}_{i}$ be the unit vector parallel to $U_{i}^{\prime}$, so that $U_{i}^{\prime}=v_{i} \tilde{r}_{i}$ for some scalar $v_{i}$. One can show that $\tilde{r}_{i}$ remains close to the eigenvector $r_{i}(u)$ of the Jacobian matrix $A(u) \doteq D f(u)$, but $\tilde{r}_{i} \neq r_{i}(u)$ in general. The first equation in (1.12) now yields the decomposition

$$
u_{x}=\sum_{i} v_{i} \tilde{r}_{i} .
$$

If $u=u(t, x)$ is a solution of (1.9), we can think of $v_{i}$ as the density of $i$-waves in $u$. The remarkable fact is that these components satisfy a system of evolution equations

$$
v_{i, t}+\left(\tilde{\lambda}_{i} v_{i}\right)_{x}-v_{i, x x}=\phi_{i} \quad i=1, \ldots, n,
$$

where the source terms $\phi_{i}$ on the right hand side are INTEGRABLE over the whole domain $\{x \in \mathbb{R}, t>0\}$. Indeed, we can think of the sources $\phi_{i}$ as the new waves produced by interactions between viscous waves. Their total strength is controlled by means of viscous interaction functionals, somewhat similar to the one introduced by Glimm in the hyperbolic case. Since the left hand side of (1.14) is in conservation form and the vectors $\tilde{r}_{i}$ have unit length, we obtain the bound

$$
\left\|u_{x}(\tau)\right\|_{\mathbf{L}^{1}} \leq \sum_{i}\left\|v_{i}(\tau)\right\|_{\mathbf{L}^{1}} \leq \sum_{i}\left(\left\|v_{i}(0)\right\|+\int_{0}^{\tau} \int\left|\phi_{i}(t, x)\right| d x d t\right) .
$$

This line of argument yields global BV bounds and stability estimates for viscous solutions. In turn, letting $\varepsilon \rightarrow 0$ in (1.8), a standard compactness argument yields the convergence of $u^{\varepsilon}$ to a weak solution $u$ of (1.1).

The plan of these notes is as follows. In Section 2 we review some basic facts about shock and rarefaction waves, and describe the solution of the Riemann problem. Section 3 is concerned with solutions of the more general Cauchy problem, obtained as limits of Glimm or front tracking approximations. Stability and uniqueness issues are discussed in the next two sections. The remaining sections 6 to 8 describe the vanishing viscosity approach, based on decomposition along gradients of viscous travelling waves and the new Lyapunov functionals controlling the interactions among viscous waves. Some further research directions are discussed in Section 9 .

\section{The Riemann problem}

Here and in the next section, we shall consider a strictly hyperbolic system of conservation laws satisfying the additional hypothesis

(H) For each $i=1, \ldots, n$, the $i$-th field is either genuinely nonlinear, so that $D \lambda_{i}(u) \cdot r_{i}(u)>0$ for all $u$, or linearly degenerate, with $D \lambda_{i}(u) \cdot r_{i}(u)=0$ for all $u$.

Notice that, in the genuinely nonlinear case, the $i$-th eigenvalue $\lambda_{i}$ is required to be strictly increasing along each integral curve of the corresponding field of 
eigenvectors $r_{i}$. In the linearly degenerate case, on the other hand, the eigenvalue $\lambda_{i}$ is constant along each such curve.

\section{Shocks and Admissibility Conditions.}

The simplest type of discontinuous solution is given by a shock :

$$
U(t, x)=\left\{\begin{array}{lll}
u^{+} & \text {if } & x>\lambda t, \\
u^{-} & \text {if } & x<\lambda t,
\end{array}\right.
$$

for some left and right states $u^{-}, u^{+} \in \mathbb{R}^{n}$ and a speed $\lambda \in \mathbb{R}$. Using the divergence theorem, one checks that the identity (1.4) is satisfied if and only if the following Rankine-Hugoniot conditions hold:

$$
\lambda\left(u^{+}-u^{-}\right)=f\left(u^{+}\right)-f\left(u^{-}\right) .
$$

Denote by $A(u)=D f(u)$ the $n \times n$ Jacobian matrix of $f$ at $u$. For any $u, v$, define the averaged matrix

$$
A(u, v) \doteq \int_{0}^{1} A(\theta u+(1-\theta) v) d \theta
$$

and call $\lambda_{i}(u, v), i=1, \ldots, n$ its eigenvalues. We can then write (2.2) in the equivalent form

$$
\lambda\left(u^{+}-u^{-}\right)=\int_{0}^{1} D f\left(\theta u^{+}+(1-\theta) u^{-}\right) \cdot\left(u^{+}-u^{-}\right) d \theta=A\left(u^{+}, u^{-}\right) \cdot\left(u^{+}-u^{-}\right) .
$$

In other words, the Rankine-Hugoniot conditions hold if and only if the jump $u^{+}-$ $u^{-}$is an eigenvector of the averaged matrix $A\left(u^{+}, u^{-}\right)$and the speed $\lambda$ coincides with the corresponding eigenvalue.

Definition 1. A function $u=u(t, x)$ has an approximate jump discontinuity at the point $(\tau, \xi)$ if there exists vectors $u^{+} \neq u^{-}$and a speed $\lambda$ such that

$$
\lim _{r \rightarrow 0+} \frac{1}{r^{2}} \int_{-r}^{r} \int_{-r}^{r}|u(\tau+t, \xi+x)-U(t, x)| d x d t=0,
$$

with $U$ as in (2.1). We say that $u$ is approximately continuous at the point $(\tau, \xi)$ if the above relations hold with $u^{+}=u^{-}$(and $\lambda$ arbitrary).

More generally, if $u$ is now a solution of the system of conservation laws (1.1) having an approximate jump, one can prove that the states $u^{-}, u^{+}$and the speed $\lambda$ again satisfy the Rankine-Hugoniot conditions.

In order to achieve uniqueness of solutions to initial value problems, it is customary to supplement the conservation equations (1.1) with additional admissibility conditions, to be satisfied at points of jump. We recall here two basic approaches.

Definition 2. A continuously differentiable function $\eta: \mathbb{R}^{n} \mapsto \mathbb{R}$ is called an entropy for the system of conservation laws (1.1), with entropy flux $q: \mathbb{R}^{n} \mapsto \mathbb{R}$, if it satisfies the identity

$$
D \eta(u) \cdot D f(u)=D q(u)
$$


An immediate consequence of (2.4) is that, if $u=u(t, x)$ is a $\mathcal{C}^{1}$ solution of (2.1), then

$$
\eta(u)_{t}+q(u)_{x}=0 .
$$

In other words, for a smooth solution $u$, not only the quantities $u_{1}, \ldots, u_{n}$ are conserved, but the additional conservation law (2.5) holds as well. However one should be aware that, for a discontinuous solution $u$, the quantity $\eta(u)$ may not be conserved.

A standard admissibility condition for weak solutions can now be formulated as follows.

Definition 3. Let $\eta$ be a convex entropy for the system (1.1), with entropy flux $q$. A weak solution $u$ is entropy-admissible if

$$
\eta(u)_{t}+q(u)_{x} \leq 0
$$

in distribution sense, i.e.

$$
\iint\left\{\eta(u) \varphi_{t}+q(u) \varphi_{x}\right\} d x d t \geq 0
$$

for every function $\varphi \geq 0$, continuously differentiable with compact support.

In analogy with (2.3), if $u$ is an entropy admissible solution, at every point of approximate jump one can show that

$$
\lambda\left[\eta\left(u^{+}\right)-\eta\left(u^{-}\right)\right] \geq q\left(u^{+}\right)-q\left(u^{-}\right) .
$$

An alternative admissibility condition, due to Lax [Lx], is particularly useful because it can be applied to any system and has a simple geometrical interpretation. We recall that, at a point of approximate jump, the speed $\lambda$ must be an eigenvalue of the averaged matrix $A\left(u^{-}, u^{+}\right)$at (2.3), i.e. $\lambda=\lambda_{i}\left(u^{-}, u^{+}\right)$for some $i \in\{1, \ldots, n\}$.

Definition 4. A solution $u=u(t, x)$ of (1.1) satisfies the Lax admissibility condition if, at each point of approximate jump, the left and right states $u^{-}, u^{+}$and the speed $\lambda=\lambda_{i}\left(u^{-}, u^{+}\right)$of the jump satisfy

$$
\lambda_{i}\left(u^{-}\right) \geq \lambda \geq \lambda_{i}\left(u^{+}\right)
$$

To appreciate the geometric meaning of this condition, consider a piecewise smooth solution, having a discontinuity along the line $x=\gamma(t)$, where the solution jumps from a left state $u^{-}$to a right state $u^{+}$. According to (2.3), this discontinuity must travel with a speed $\lambda \doteq \dot{\gamma}=\lambda_{i}\left(u^{-}, u^{+}\right)$equal to an eigenvalue of the averaged matrix $A\left(u^{-}, u^{+}\right)$. If we now look at the $i$-characteristics, i.e. at the solutions of the O.D.E.

$$
\dot{x}=\lambda_{i}(u(t, x))
$$

we see that the Lax condition (2.9) requires that these lines run into the shock, from both sides.

\section{Centered Rarefaction Waves.}

Next, we consider another special type of solutions, in the form of centered rarefaction waves. Fix a state $u_{0} \in \mathbb{R}^{n}$ and an index $i \in\{1, \ldots, n\}$. As before, let $r_{i}(u)$ be an $i$-eigenvector of the Jacobian matrix $A(u)=D f(u)$. The integral curve 
of the vector field $r_{i}$ through the point $u_{0}$ is called the $i$-rarefaction curve through $u_{0}$. It is obtained by solving the Cauchy problem in state space:

$$
\frac{d u}{d \sigma}=r_{i}(u), \quad u(0)=u_{0} .
$$

We shall denote this curve as

$$
\sigma \mapsto R_{i}(\sigma)\left(u_{0}\right) .
$$

Clearly, the parametrization depends on the choice of the eigenvectors $r_{i}$. In particular, if we impose the normalization $\left|r_{i}(u)\right| \equiv 1$, then the rarefaction curve $R_{i}$ will be parametrized by arc-length.

Let the $i$-th field be genuinely nonlinear, and assume that $u^{+}$lies on the positive $i$-rarefaction curve through $u^{-}$, i.e. $u^{+}=R_{i}(\sigma)\left(u^{-}\right)$for some $\sigma>0$. For each $s \in[0, \sigma]$, define

$$
\lambda_{i}(s)=\lambda_{i}\left(R_{i}(s)\left(u^{-}\right)\right) .
$$

Observe that, by genuine nonlinearity, the map $s \mapsto \lambda_{i}(s)$ is strictly increasing. Hence, for every $\lambda \in\left[\lambda_{i}\left(u^{-}\right), \lambda_{i}\left(u^{+}\right)\right]$, there is a unique value $s \in[0, \sigma]$ such that $\lambda=\lambda_{i}(s)$. We claim that, for $t>0$, the function

$$
u(t, x)=\left\{\begin{array}{lll}
u^{-} & \text {if } & x / t<\lambda_{i}\left(u^{-}\right), \\
R_{i}(s)\left(u^{-}\right) & \text {if } & x / t=\lambda_{i}(s) \in\left[\lambda_{i}\left(u^{-}\right), \lambda_{i}\left(u^{+}\right)\right], \\
u^{+} & \text {if } \quad \mathrm{x} / \mathrm{t}>\lambda_{i}\left(u^{+}\right),
\end{array}\right.
$$

is a continuous solution of the Riemann problem (1.1), (1.7). Indeed, by construction it follows

$$
\lim _{t \rightarrow 0+}\|u(t, \cdot)-\bar{u}\|_{\mathbf{L}^{1}}=0 .
$$

Moreover, the equation (1.3) is trivially satisfied in the sectors where $x / t<\lambda_{i}\left(u^{-}\right)$ or $x / t>\lambda_{i}\left(u^{+}\right)$, since here $u_{t}=u_{x}=0$. Next, assume $x / t=\lambda_{i}(s)$ for some $s \in] 0, \sigma[$. Since $u$ is constant along each ray through the origin $\{x / t=c\}$, we have

$$
u_{t}(t, x)+\frac{x}{t} u_{x}(t, x)=0
$$

We now observe that the definition (2.11) implies $x / t=\lambda_{i}(u(t, x))$. Moreover, $u_{x}$ is parallel to $r_{i}(u)$, hence it is an eigenvector of the Jacobian matrix $A(u)$ with eigenvalue $\lambda_{i}(u)$. On the sector $\left\{\lambda_{i}\left(u^{-}\right)<x / t<\lambda_{i}\left(u^{+}\right)\right\}$we thus have $u_{t}+A(u) u_{x}=0$, proving our claim. Notice that the assumption $\sigma>0$ is essential for the validity of this construction. In the opposite case $\sigma<0$, the definition (2.11) would yield a triple-valued function in the region where $x / t \in] \lambda_{i}\left(u^{+}\right), \lambda_{i}\left(u^{-}\right)[$.

\section{Solution of the Riemann Problem.}

Relying on the previous analysis, the solution of the general Riemann problem

$$
u_{t}+f(u)_{x}=0, \quad u(0, x)= \begin{cases}u^{-} & \text {if } x<0, \\ u^{+} & \text {if } x>0 .\end{cases}
$$

can be obtained by finding intermediate states $\omega_{0}=u^{-}, \omega_{1}, \ldots, \omega_{n}=u^{+}$such that each pair of adiacent states $\omega_{i-1}, \omega_{i}$ can be connected by an $i$-shock, or by a centered rarefaction $i$-wave. By the implicit function theorem, this can always be done 


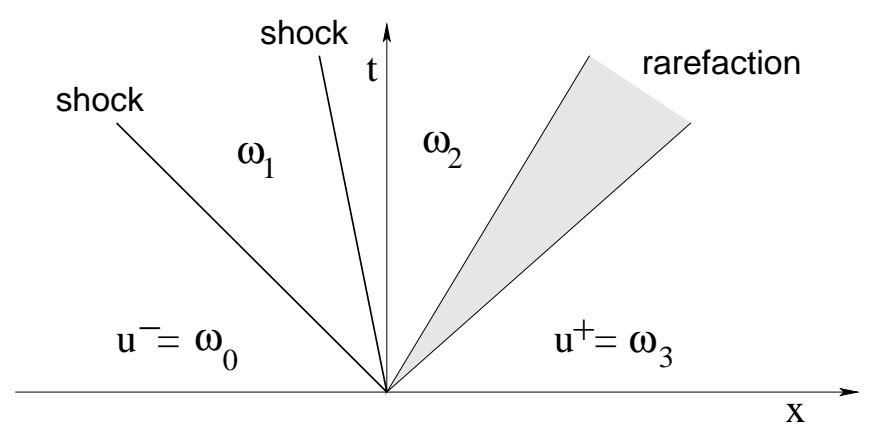

figure 3

provided that the two states $u^{-}, u^{+}$are sufficiently close. The complete solution is now obtained by piecing together the solutions of the $n$ Riemann problems

$$
u_{t}+f(u)_{x}=0, \quad u(0, x)=\left\{\begin{array}{lll}
\omega_{i-1} & \text { if } & x<0, \\
\omega_{i} & \text { if } & x>0,
\end{array}\right.
$$

on different sectors of the $t-x$ plane. A typical solution is illustrated in fig. 3 .

\section{Glimm and front tracking approximations}

Approximate solutions to a more general Cauchy problem can be constructed by patching together solutions of several Riemann problems. In the Glimm scheme (fig. 4), one works with a fixed grid in the $t-x$ plane, with mesh sizes $\Delta t, \Delta x$. At time $t=0$ the initial data is approximated by a piecewise constant function, with jumps at grid points. Solving the corresponding Riemann problems, a solution is constructed up to a time $\Delta t$ sufficiently small so that waves emerging from different nodes do not interact. At time $t_{1} \doteq \Delta t$, we replace the solution $u(\Delta t, \cdot)$ by a piecewise constant function having jumps exactly at grid points. Solving the new Riemann problems at every one of these points, one can prolong the solution to the next time interval $[\Delta t, 2 \Delta t]$. At time $t_{2} \doteq 2 \Delta t$, the solution is a gain approximated by a piecewise constant functions with jumps exactly at grid points, etc... A key ingredient of the Glimm scheme is the restarting procedure. At each time $t_{j} \doteq j \Delta t$, a natural way to approximate a BV function with a piecewise constant one is by taking its average value on each subinterval $J_{i} \doteq\left[x_{i-1}, x_{i}\right]$. However, this procedure may generate an arbitrarily large amount of oscillations. Instead, the Glimm scheme is based on random sampling: a point $y_{i}$ is selected at random inside each interval $J_{i}$ and the value $u\left(t_{j}-, y_{i}\right)$ is taken as the new value of $u\left(t_{j}, x\right)$ for all $x \in J_{i}$. An excellent introduction to the Glimm scheme can be found in the book by J. Smoller [Sm].

An alternative technique for contructing approximate solutions is by wave-front tracking (fig. 5). This method was introduced by Dafermos [D1] in the scalar case and later developed by various authors [DP1], [B1], [Ri], [BJ]. It now provides an 


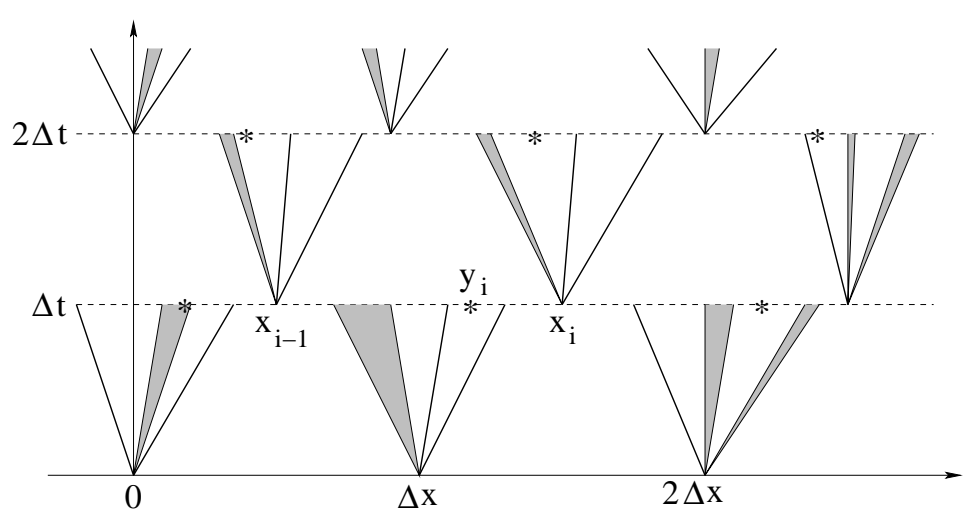

figure 4

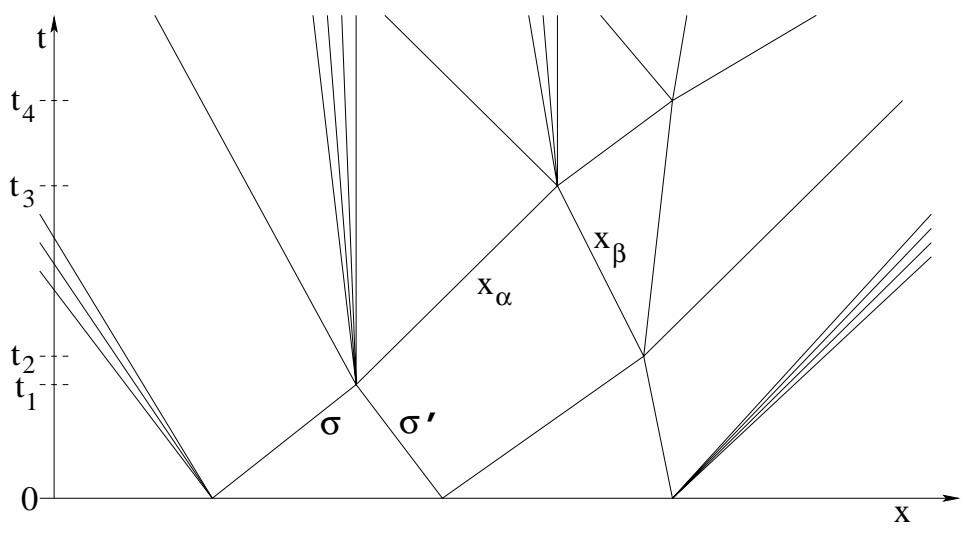

figure 5

efficient tool in the study of general $n \times n$ systems of conservation laws, both for theoretical and numerical purposes [B3], [HR].

The initial data is here approximated with a piecewise constant function, and each Riemann problem is solved approximately, within the class of piecewise constant functions. In particular, if the exact solution contains a centered rarefaction, this must be approximated by a rarefaction fan, containing several small jumps. At the first time $t_{1}$ where two fronts interact, the new Riemann problem is again approximately solved by a piecewise constant function. The solution is then prolonged up to the second interaction time $t_{2}$, where the new Riemann problem is solved, etc... The main difference is that in the Glimm scheme one specifies a priori the nodal points where the the Riemann problems are to be solved. On the other hand, in a solution constructed by wave-front tracking the locations of the jumps and of the interaction points depend on the solution itself. No restarting procedure is needed and the map $t \mapsto u(t, \cdot)$ is thus continuous with values in $\mathbf{L}_{\text {loc }}^{1}$. 
In the end, both algorithms produce a sequence of approximate solutions, whose convergence relies on a compactness argument based on uniform bounds on the total variation. We sketch the main idea involved in these a priori BV bounds. Consider a piecewise constant function $u: \mathbb{R} \mapsto \mathbb{R}^{n}$, say with jumps at points $x_{1}<x_{2}<\cdots<x_{N}$. Call $\sigma_{\alpha}$ the amplitude of the jump at $x_{\alpha}$. The total strength of waves is then defined as

$$
V(u) \doteq \sum_{\alpha}\left|\sigma_{\alpha}\right|,
$$

Clearly, this is an equivalent way to measure the total variation. Along a solution $u=u(t, x)$ constructed by front tracking, the quantity $V(t)=V(u(t, \cdot))$ may well increase at interaction times. To provide global a priori bounds, following [G] one introduces a wave interaction potential, defined as

$$
Q(u)=\sum_{(\alpha, \beta) \in \mathcal{A}}\left|\sigma_{\alpha} \sigma_{\beta}\right|,
$$

where the summation runs over the set $\mathcal{A}$ of all couples of approaching waves. Roughly speaking, we say that two wave-fronts located at $x_{\alpha}<x_{\beta}$ are approaching if the one at $x_{\alpha}$ has a faster speed than the one at $x_{\beta}$ (hence the two fronts are expected to collide at a future time). Now consider a time $\tau$ where two incoming wave-fronts interact, say with strengths $\sigma, \sigma^{\prime}$ (for example, take $\tau=t_{1}$ in fig. 5). The difference between the outgoing waves emerging from the interaction and the two incoming waves $\sigma, \sigma^{\prime}$ is of magnitude $\mathcal{O}(1) \cdot\left|\sigma \sigma^{\prime}\right|$. On the other hand, after time $\tau$ the two incoming waves are no longer approaching. This accounts for the decrease of the functional $Q$ in (3.2) by the amount $\left|\sigma \sigma^{\prime}\right|$. Observing that the new waves generated by the interaction could approach all other fronts, the change in the functionals $V, Q$ across the interaction time $\tau$ is estimated as

$$
\Delta V(\tau)=\mathcal{O}(1) \cdot\left|\sigma \sigma^{\prime}\right|, \quad \Delta Q(\tau)=-\left|\sigma \sigma^{\prime}\right|+\mathcal{O}(1) \cdot\left|\sigma \sigma^{\prime}\right| V(\tau-) .
$$

If the initial data has small total variation, for a suitable constant $C_{0}$ the quantity

$$
\Upsilon(t) \doteq V(u(t, \cdot))+C_{0} Q(u(t, \cdot))
$$

is monotone decreasing in time. This argument provides the uniform BV bounds on all approximate solutions. Using Helly's compactness theorem, one obtains the convergence of a subsequence of approximate solutions, and hence the global existence of a weak solution.

Theorem 1. Let the system (1.1) be strictly hyperbolic and satisfy the assumptions (H). Then, for a sufficiently small $\delta>0$ the following holds. For every initial condition $\bar{u}$ with

$$
\|\bar{u}\|_{\mathbf{L}^{\infty}}<\delta, \quad \quad \text { Tot. Var. }\{\bar{u}\}<\delta,
$$

the Cauchy problem has a weak solution, defined for all times $t \geq 0$.

The proof is based on careful analysis of solutions of the Riemann problem and on the use of a quadratic interaction functional (3.2) to control the creation of new waves. These techniques also provided the basis for subsequent investigations of Glimm and Lax [GL] and Liu [L2], [L3] on the asymptotic behavior of weak solutions as $t \rightarrow \infty$. 


\section{Stability}

The previous existence result relied on a compactness argument which, by itself, does not provide informations on the uniqueness of solutions. A first understanding of the dependence of weak solutions on the initial data was provided by the analysis of front tracking approximations. The idea is to perturb the initial data by shifting the position of one of the jumps, say from $x$ to a nearby point $x^{\prime}$. By carefully estimating the corresponding shifts in the positions of all wave-fronts at a later time $t$, one obtains a bound on the $\mathbf{L}^{1}$ distance between the original and the perturbed approximate solution. After much technical work, this approach yielded a proof of the Lipschitz continuous dependence of solutions on the initial data, first in [BC1] for $2 \times 2$ systems, then in [BCP] for general $n \times n$ systems.

Theorem 2. Let the system (1.1) be strictly hyperbolic and satisfy the assumptions (H). Then, for every initial data $\bar{u}$ satisfying (3.3) the weak solution obtained as limit of front tracking approximations is unique and depends Lipschitz continuously on the initial data, in the $\mathbf{L}^{1}$ distance.

These weak solutions can thus be written in the form $u(t, \cdot)=S_{t} \bar{u}$, as trajectories of a semigroup $S: \mathcal{D} \times[0, \infty[\mapsto \mathcal{D}$ on some domain $\mathcal{D}$ containing all functions with sufficiently small total variation. For some Lipschitz constants $L, L^{\prime}$ one has

$$
\left\|S_{t} \bar{u}-S_{s} \bar{v}\right\|_{\mathbf{L}^{1}} \leq L\|\bar{u}-\bar{v}\|_{\mathbf{L}^{1}}+L^{\prime}|t-s|
$$

for all $t, s \geq 0$ and initial data $\bar{u}, \bar{v} \in \mathcal{D}$.

A more transparent proof of Theorem 2 was later achieved by a technique introduced by Liu and Yang in [LY] and developed in [BLY] into its final form. The heart of the matter is to construct a nonlinear functional, equivalent to the $\mathbf{L}^{1}$ distance, which is decreasing in time along every pair of solutions. We thus seek $\Phi=\Phi(u, v)$ and a constant $C$ such that

$$
\begin{gathered}
\frac{1}{C} \cdot\|v-u\|_{\mathbf{L}^{1}} \leq \Phi(u, v) \leq C \cdot\|v-u\|_{\mathbf{L}^{1}}, \\
\frac{d}{d t} \Phi(u(t), v(t)) \leq 0 .
\end{gathered}
$$

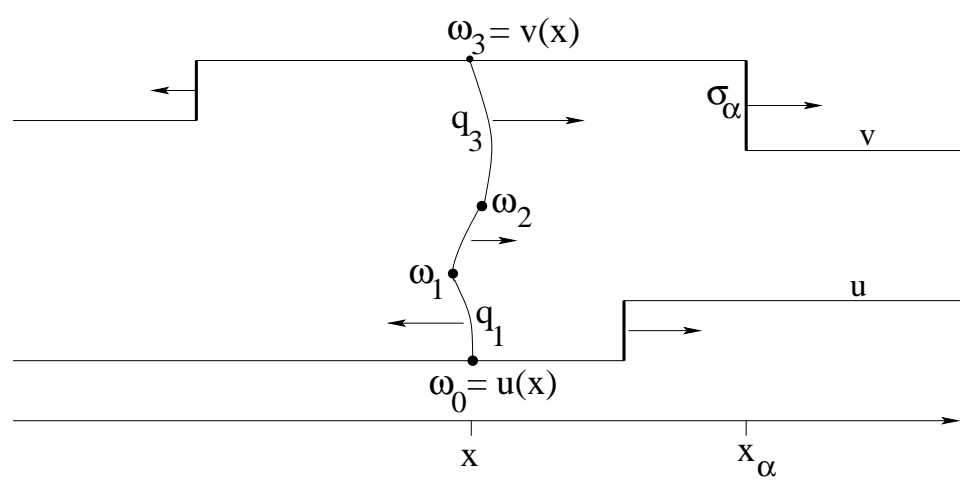

figure 6 
Throughout the following, it will be convenient to measure the strength of wavefronts of a genuinely nonlinear family in terms of the change in the characteristic speed. If $u$ has a jump in the $k$-th family at the point $x_{\alpha}$, in the genuinely nonlinear case we thus define the strength as

$$
\sigma_{\alpha} \doteq \lambda_{k}\left(u\left(x_{\alpha}+\right)\right)-\lambda_{k}\left(u\left(x_{\alpha}-\right)\right) .
$$

In connection with piecewise constant functions $u, v: \mathbb{R} \mapsto \mathbb{R}^{n}$ generated by a front tracking algorithm, the functional $\Phi$ can be defined as follows (fig. 6). At each point $x$, we connect the states $u(x), v(x)$ by means of $n$ shock curves. In other words, we construct intermediate states $\omega_{0}=u(x), \omega_{1}, \ldots, \omega_{n}=v(x)$ such that each pair $\omega_{i-1}, \omega_{i}$ is connected by an $i$-shock. These states can be uniquely determined by the implicit function theorem. Call $q_{1}, \ldots, q_{n}$, the strengths of these shocks. We regard $q_{i}(x)$ as the $i$-th scalar component of the jump $(u(x), v(x))$. For some constant $C^{\prime}$, one clearly has

$$
\frac{1}{C^{\prime}} \cdot|v(x)-u(x)| \leq \sum_{i=1}^{n}\left|q_{i}(x)\right| \leq C^{\prime} \cdot|v(x)-u(x)| .
$$

The functional $\Phi$ is now defined as

$$
\Phi(u, v) \doteq \sum_{i=1}^{n} \int_{-\infty}^{\infty} W_{i}(x)\left|q_{i}(x)\right| d x
$$

where the weights $W_{i}$ take the form

$$
\begin{aligned}
W_{i}(x) \doteq 1 \quad & +\kappa_{1} \cdot\left[\text { total strength of waves in } u \text { and in } v \text { which approach the } i \text {-wave } q_{i}(x)\right] \\
& +\kappa_{2} \cdot[\text { wave interaction potentials of } u \text { and of } v] \\
\doteq 1 \quad & +\kappa_{1} V_{i}(x)+\kappa_{2}[Q(u)+Q(v)]
\end{aligned}
$$

for suitable constants $\kappa_{1}, \kappa_{2}$. Notice that, by construction, $q_{i}(x)$ represents the strength of a fictitious shock wave located at $x$, travelling with a speed $\lambda_{i}(x)$ determined by the Rankine-Hugoniot equations. In (4.7), it is thus meaningful to consider the quantity

$$
V_{i}(x) \doteq \sum_{\alpha \in \mathcal{A}_{i}(x)}\left|\sigma_{\alpha}\right|,
$$

where the summation extends to all wave-fronts $\sigma_{\alpha}$ in $u$ and in $v$ which are approaching the $i$-shock $q_{i}(x)$. By (4.5) and the boundedness of the weights $W_{i}$, it is clear that (4.2) holds.

Next, we will sketch an argument showing why the functional $\Phi$ should decrease along pairs of solutions. For a detailed proof, see [BLY] or [B3]. We first observe that the maps $t \mapsto q_{i}(t, \cdot)$ are Lipschitz continuous as maps with values into $\mathbf{L}^{1}$. At a time $\tau$ where an interaction occurs in $u$ or in $v$, if the coefficient $\kappa_{2}$ is sufficiently large the decrease of the functionals $Q(u)$ or $Q(v)$ implies

$$
W_{i}(\tau+, x)<W_{i}(\tau-, x),
$$

for every $i \in\{1, \ldots, n\}$ and almost every $x \in \mathbb{R}$. Hence $\Phi$ is decreasing at each interaction time. 


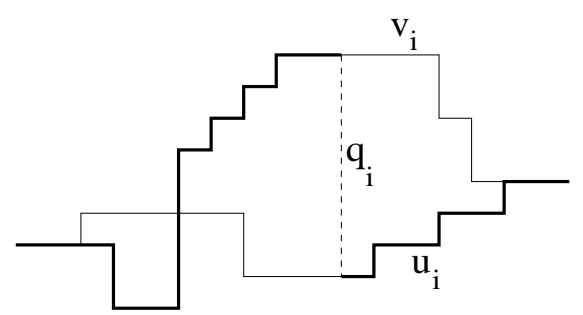

figure 7

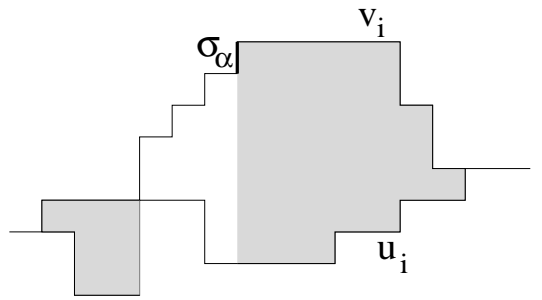

figure 8

Interchanging the order of the summation and the integral, the functional $\Phi$ in (4.6)-(4.7) can be written in the equivalent form

$$
\begin{aligned}
\Phi(u, v)=[1 & \left.+\kappa_{2} Q(u)+\kappa_{2} Q(v)\right] \cdot \sum_{i=1}^{n} \int_{-\infty}^{\infty}\left|q_{i}(x)\right| d x \\
& +\kappa_{1} \cdot \sum_{\sigma_{\alpha} \in \mathcal{J}(u) \cup \mathcal{J}(v)}\left|\sigma_{\alpha}\right| \cdot \sum_{i} \int_{q_{i}(x) \text { approaches } \sigma_{\alpha}}\left|q_{i}(x)\right| d x .
\end{aligned}
$$

For our further analysis, the following construction will be useful. Given two piecewise constant functions $u, v$ with compact support, for $i=1, \ldots, n$ we can define the scalar components $u_{i}, v_{i}$ by induction on the jump points of $u, v$. We start by setting $u_{i}(-\infty)=v_{i}(-\infty)=0$. If $x_{\alpha} \in \mathcal{J}(u)$ is a jump point of $u$, then we let $v_{i}$ be constant across $x_{\alpha}$ and set

$$
u_{i}\left(x_{\alpha}+\right) \doteq u_{i}\left(x_{\alpha}-\right)-\left[q_{i}\left(x_{\alpha}+\right)-q_{i}\left(x_{\alpha}-\right)\right] .
$$

On the other hand, if $x_{\alpha} \in \mathcal{J}(v)$ is a jump point of $v$, then we let $u_{i}$ be constant across $x_{\alpha}$ and set

$$
v_{i}\left(x_{\alpha}+\right) \doteq v_{i}\left(x_{\alpha}-\right)+\left[q_{i}\left(x_{\alpha}+\right)-q_{i}\left(x_{\alpha}-\right)\right] .
$$

These definitions trivially imply

$$
q_{i}(x)=v_{i}(x)-u_{i}(x) \quad \text { for all } x \in \mathbb{R}, i=1, \ldots, n .
$$

By definition, the $i$-waves in $u$ and $v$ which approach $q_{i}(x)$ are those located within the thick portions of the graphs of $u_{i}, v_{i}$ in fig. 7. Viceversa, for a given $i$-wave $\sigma_{\alpha}$ located at $x_{\alpha}$, the regions where the jumps $q_{i}(x)$ approach $\sigma_{\alpha}$ are represented by the shaded areas in in fig. 8.

To understand why the expression (4.8) decreases in time, assume first that $v$ has a wave-front at $x_{\alpha}$ with strength $\sigma_{\alpha}$, say in the $k$-th family. In connection with this front (fig. 9), for every $i<k$ the functional $\Phi(u, v)$ contains a term of the form $\Phi_{\alpha, i} \doteq \kappa_{1} \cdot\left|\sigma_{\alpha}\right| \cdot\left[\right.$ area of the region between the graphs of $u_{i}$ and $v_{i}$, to the right of $x_{\alpha}$ ].

By strict hyperbolicity, the $i$-th and $k$-th characteristic speeds are strictly separated, say $\lambda_{k}-\lambda_{i} \geq c>0$. We thus expect

$$
\frac{d \Phi_{\alpha, i}}{d t} \approx-\kappa_{1}\left|\sigma_{\alpha}\right|\left|q_{i}^{\alpha}\right|\left(\dot{x}_{\alpha}-\lambda_{i}^{\alpha}\right) \leq-c \kappa_{1}\left|\sigma_{\alpha}\right|\left|q_{i}^{\alpha}\right|
$$

up to higher order terms. Here $\lambda_{i}^{\alpha} \doteq \lambda_{i}\left(\omega_{i-1}\left(x_{\alpha}\right), \omega_{i}\left(x_{\alpha}\right)\right)$ is the speed of the $i$-shock $q_{i}^{\alpha}$. In addition, for every $i>k$ the functional $\Phi(u, v)$ contains a term of the form

$\Phi_{\alpha, i} \doteq \kappa_{1} \cdot\left|\sigma_{\alpha}\right| \cdot\left[\right.$ area of the region between the graphs of $u_{i}$ and $v_{i}$, to the left of $\left.x_{\alpha}\right]$.

Entirely similar estimates can be proved also for these terms. 


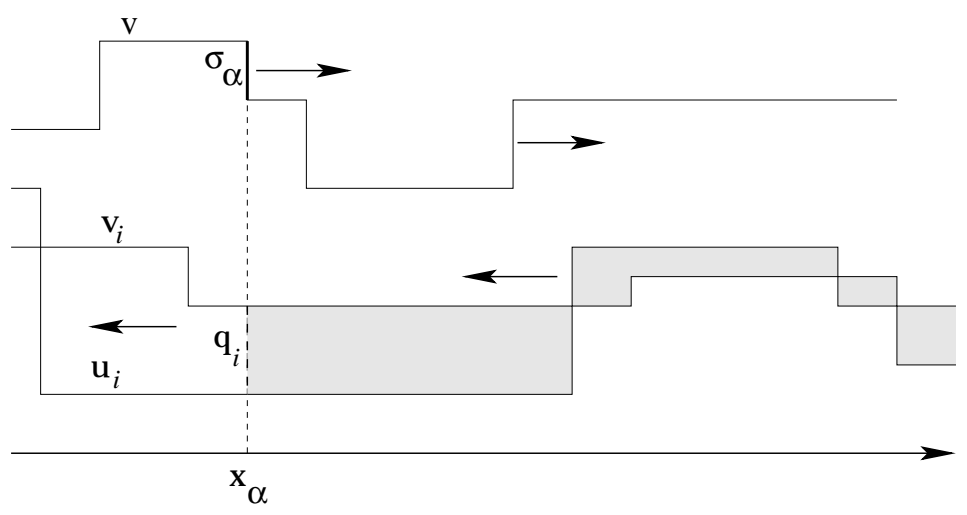

figure 9

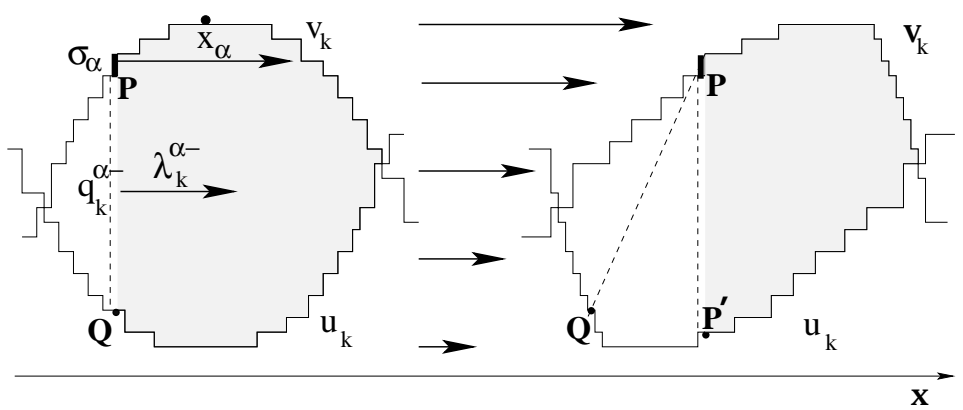

figure 10

Next, if the $k$-th field is genuinely nonlinear, the functional $\Phi(u, v)$ also contains a term of the form (fig. 10)

$\Phi_{\alpha, k} \doteq \kappa_{1} \cdot\left|\sigma_{\alpha}\right| \cdot\left[\right.$ area of the region between the graphs of $u_{k}$ and $v_{k}$, to the right of $\left.x_{\alpha}\right]$.

Notice that, because of genuine nonlinearity, the points on the graphs of $u_{k}$ and $v_{k}$ move with different speeds. As a consequence, the shape of these graphs changes in time. In particular, while the area enclosed by the two graphs may remain constant, the portion of this area located to the right of a given front $\sigma_{\alpha}$ will decrease. In figure 10, the two points $P, Q$ lie initially on the same vertical line. At a later time this is no longer true. The area of the shaded region, enclosed by the graphs of $u_{k}$ and $v_{k}$ and by a vertical line through $P$, has decreased by an amount roughly equal to the area of the triangular region with vertices $P, P^{\prime}, Q$.

The difference between the speed $\dot{x}_{\alpha}$ of the jump $\sigma_{\alpha}$ at $P$ and the average speed $\lambda_{k}^{\alpha-}$ of points between $P$ and $Q$ is estimated by

$$
\dot{x}_{\alpha}-\lambda_{k}^{\alpha-} \approx \frac{1}{2}\left|q_{k}^{\alpha-}+\sigma_{\alpha}\right| .
$$




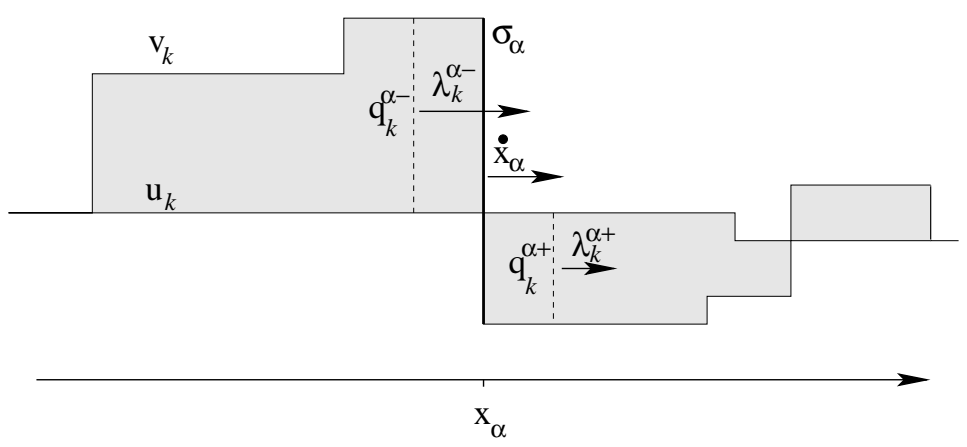

figure 11

Hence, up to higher order terms, the time derivative of $\Phi_{\alpha, k}$ satisfies

$$
\frac{d \Phi_{\alpha, k}}{d t} \approx-\kappa_{1}\left|\sigma_{\alpha}\right|\left|q_{k}^{\alpha-}\right|\left(\dot{x}_{\alpha}-\lambda_{k}^{\alpha-}\right) \approx-\kappa_{1}\left|\sigma_{\alpha}\right|\left|q_{k}^{\alpha-}\right| \cdot \frac{1}{2}\left|q_{k}^{\alpha-}+\sigma_{\alpha}\right|
$$

Yet another kind of estimate is needed in the case where the jump in $v_{k}$ crosses the graph of $u_{k}$, say $v_{k}\left(x_{\alpha}+\right)<u_{k}\left(x_{\alpha}\right)<v_{k}\left(x_{\alpha}-\right)$. In this case, the estimates (4.9) remain valid. In connection with the $k$-th field, the functional $\Phi$ contains a term of the form

$$
\Phi_{k}^{*} \doteq 1 \cdot\left[\text { area of the region between the graphs of } u_{k} \text { and } v_{k}\right]
$$

where the above area includes points both on the right and on the left of $x_{\alpha}$.

For convenience, call $\bar{\lambda}_{k} \doteq \lambda_{k}\left(v\left(x_{\alpha}+\right)\right)$. Since $q_{k}^{\alpha+} \approx q_{k}^{\alpha-}+\sigma_{\alpha}$, the genuine nonlinearity of the $k$-th characteristic field and the parametrization (4.4) yield

$$
\begin{gathered}
\lambda_{k}^{\alpha-} \approx \bar{\lambda}_{k}+\left|q_{k}^{\alpha+}\right|+\frac{1}{2}\left|q_{k}^{\alpha-}\right| \quad \lambda_{k}^{\alpha+} \approx \bar{\lambda}_{k}+\frac{1}{2}\left|q_{k}^{\alpha+}\right|, \\
\dot{x}_{\alpha} \approx \bar{\lambda}_{k}+\frac{1}{2}\left(\left|q_{k}^{\alpha+}\right|+\left|q_{k}^{\alpha-}\right|\right) .
\end{gathered}
$$

Neglegting higher order terms, the shaded area in fig. 11 thus decreases at the rate

$$
\frac{d \Phi_{k}^{*}}{d t} \approx\left|q_{k}^{\alpha-}\right|\left(\lambda_{k}^{\alpha-}-\dot{x}_{\alpha}\right)+\left|q_{k}^{\alpha+}\right|\left(\dot{x}_{\alpha}-\lambda_{k}^{\alpha+}\right) \approx\left|q_{k}^{\alpha-}\right|\left|q_{k}^{\alpha+}\right| \approx\left|q_{k}^{\alpha-}\right|\left|\sigma_{\alpha}+q_{k}^{\alpha-}\right| .
$$

Notice that in this last case the decrease of the functional $\Phi$ is simply due to the decrease of the $\mathbf{L}^{1}$ distance between the $k$-th components of $u$ and $v$. In this occasion, the weights $W_{k}$ play no role. For all details we always refer to [B3].

For general $n \times n$ systems, in (4.1) one finds a Lipschitz constant $L>1$. Indeed, it is only in the scalar case that the semigroup is contractive and the theory of accretive operators and abstract evolution equations in Banach spaces can be applied, see $[\mathrm{K}],[\mathrm{C}]$. We refer to the flow generated by a system of conservation laws as a Riemann semigroup, because it is entirely determined by specifying how Riemann problems are solved. As proved in [B2], if two semigroups $S, S^{\prime}$ yield the same solutions to all Riemann problems, then they coincide, up to the choice of their domains. 
From (4.1) one can deduce the error bound

$$
\left\|w(T)-S_{T} w(0)\right\|_{\mathbf{L}^{1}} \leq L \cdot \int_{0}^{T}\left\{\liminf _{h \rightarrow 0+} \frac{\left\|w(t+h)-S_{h} w(t)\right\|_{\mathbf{L}^{1}}}{h}\right\} d t,
$$

valid for every Lipschitz continuous map $w:[0, T] \mapsto \mathcal{D}$ taking values inside the domain of the semigroup. We can think of $t \mapsto w(t)$ as an approximate solution of (1.1), while $t \mapsto S_{t} w(0)$ is the exact solution having the same initial data. According to (4.12), the distance at time $T$ is bounded by the integral of an instantaneous error rate, amplified by the Lipschitz constant $L$ of the semigroup.

Using (4.12), one can estimate the distance between a front tracking approximation and the corresponding exact solution. For approximate solutions constructed by the Glimm scheme, a direct application of this same formula is not possible because of the additional errors introduced by the restarting procedures at times $t_{k} \doteq k \Delta t$. However, relying on a careful analysis of Liu [L4], one can construct a front tracking approximate solution having the same initial and terminal values as the Glimm solution. By this technique, in $[\mathrm{BM}]$ the authors proved the estimate

$$
\lim _{\Delta x \rightarrow 0} \frac{\left\|u^{\mathrm{Glimm}}(\tau, \cdot)-u^{\text {exact }}(\tau, \cdot)\right\|_{\mathbf{L}^{1}}}{\sqrt{\Delta x} \cdot|\ln \Delta x|}=0
$$

for every $\tau>0$. In other words, letting the mesh sizes $\Delta x, \Delta t \rightarrow 0$ while keeping their ratio $\Delta x / \Delta t$ constant, the $\mathbf{L}^{1}$ norm of the error in the Glimm approximate solution tends to zero at a rate slightly slower than $\sqrt{\Delta x}$.

\section{Uniqueness}

The uniqueness and stability results stated in Theorem 2 refer to a special class of weak solutions: those obtained as limits of Glimm or front tracking approximations. For several applications, it is desirable to have a uniqueness theorem valid for general weak solutions, without reference to any particular constructive procedure. Results in this direction were proved in, [BLF], [BG], [BLw]. They are all based on the error formula (4.12). In the proofs, one considers a weak solution $u=u(t, x)$ of the Cauchy problem

$$
u_{t}+f(u)_{x}=0, \quad u(0, x)=\bar{u}(x) .
$$

Assuming that $u$ satisfies suitable entropy and regularity conditions, one shows that

$$
\liminf _{h \rightarrow 0+} \frac{\left\|u(t+h)-S_{h} u(t)\right\|_{\mathbf{L}^{1}}}{h}=0
$$

at almost every time $t$. By (4.12), $u$ thus coincides with the semigroup trajectory $t \mapsto S_{t} u(0)=S_{t} \bar{u}$. Of course, this implies uniqueness. As an example, we state below the result of $[\mathrm{BLw}]$. Consider the following assumptions:

(A1) (Conservation Equations) The function $u=u(t, x)$ is a weak solution of the Cauchy problem (5.1) taking values within the domain $\mathcal{D}$ of the semigroup $S$. 
More precisely, $u:[0, T] \mapsto \mathcal{D}$ is continuous w.r.t. the $\mathbf{L}^{1}$ distance. The initial condition $u(0, x)=\bar{u}(x)$ holds, together with

$$
\iint\left[u \phi_{t}+f(u) \phi_{x}\right] d x d t=0
$$

for every $\mathcal{C}^{1}$ function $\phi$ with compact support contained inside the open strip ] $0, T[\times \mathbb{R}$.

(A2) (Lax Entropy Condition) Let $u$ have an approximate jump discontinuity at some point $(\tau, \xi) \in] 0, T[\times \mathbb{R}$. In other words, assume that there exists states $u^{-}, u^{+} \in \Omega$ and a speed $\lambda \in \mathbb{R}$ such that, calling

$$
U(t, x) \doteq\left\{\begin{array}{lll}
u^{-} & \text {if } & x<\xi+\lambda(t-\tau), \\
u^{+} & \text {if } & x>\xi+\lambda(t-\tau),
\end{array}\right.
$$

there holds

$$
\lim _{\rho \rightarrow 0+} \frac{1}{\rho^{2}} \int_{\tau-\rho}^{\tau+\rho} \int_{\xi-\rho}^{\xi+\rho}|u(t, x)-U(t, x)| d x d t=0 .
$$

Then, for some $i \in\{1, \ldots, n\}$, one has the entropy inequality:

$$
\lambda_{i}\left(u^{-}\right) \geq \lambda \geq \lambda_{i}\left(u^{+}\right) .
$$

(A3) (Bounded Variation Condition) For some small constant $\delta>0$, the function $x \mapsto u(\tau(x), x)$ has bounded variation along every Lipschitz continuous space-like curve $\{t=\tau(x)\}$, such that $\left|\tau(x)-\tau\left(x^{\prime}\right)\right| \leq \delta\left|x-x^{\prime}\right|$ for all $x, x^{\prime}$.

Theorem 3. Let $u=u(t, x)$ be a weak solution of the Cauchy problem (5.1), satisfying the assumptions (A1), (A2) and (A3). Then

$$
u(t, \cdot)=S_{t} \bar{u}
$$

for all t. In particular, the solution that satisfies the three above conditions is unique.

An additional characterization of these solutions, based on local integral estimates, was given in [B2]. The underlying idea is as follows. In a forward neighborhood of a point $(\tau, \xi)$ where $u$ has a jump, the weak solution $u$ should behave much in the same way as the solution of the corresponding Riemann problem. On the other hand, on a region where its total variation is small, our solution $u$ should be accurately approximated by the solution of a linear hyperbolic system with constant coefficients.

To state the result more precisely, we introduce some notations. Given a function $u=u(t, x)$ and a point $(\tau, \xi)$, we denote by $U_{(u ; \tau, \xi)}^{\sharp}$ the solution of the Riemann problem with initial data

$$
u^{-}=\lim _{x \rightarrow \xi-} u(\tau, x), \quad u^{+}=\lim _{x \rightarrow \xi+} u(\tau, x) .
$$

In addition, we define $U_{(u ; \tau, \xi)}^{b}$ as the solution of the linear hyperbolic Cauchy problem with constant coefficients

$$
w_{t}+\widehat{A} w_{x}=0, \quad w(0, x)=u(\tau, x)
$$


Here $\widehat{A} \doteq A(u(\tau, \xi))$. Observe that (5.8) is obtained from the quasilinear system

$$
u_{t}+A(u) u_{x}=0 \quad(A=D f)
$$

by "freezing" the coefficients of the matrix $A(u)$ at the point $(\tau, \xi)$ and choosing $u(\tau)$ as initial data. A new notion of "good solution" can now be introduced, by locally comparing a function $u$ with the self-similar solution of a Riemann problem and with the solution of a linear hyperbolic system with constant coefficients. More precisely, we say that a function $u=u(t, x)$ is a viscosity solution of the system (1.1) if $t \mapsto u(t, \cdot)$ is continuous as a map with values into $\mathbf{L}_{l o c}^{1}$, and moreover the following integral estimates hold.

(i) At every point $(\tau, \xi)$, for every $\beta^{\prime}>0$ one has

$$
\lim _{h \rightarrow 0+} \frac{1}{h} \int_{\xi-\beta^{\prime} h}^{\xi+\beta^{\prime} h}\left|u(\tau+h, x)-U_{(u ; \tau, \xi)}^{\sharp}(h, x-\xi)\right| d x=0 .
$$

(ii) There exist constants $C, \beta>0$ such that, for every $\tau \geq 0$ and $a<\xi<b$, one has

$\limsup _{h \rightarrow 0+} \frac{1}{h} \int_{a+\beta h}^{b-\beta h}\left|u(\tau+h, x)-U_{(u ; \tau, \xi)}^{b}(h, x)\right| d x \leq C \cdot(\text { Tot.Var. }\{u(\tau) ;] a, b[\})^{2}$.

As proved in [B2], this concept of viscosity solution completely characterizes semigroup trajectories.

Theorem 4. Let $S: \mathcal{D} \times[0, \infty[\times \mathcal{D}$ be a semigroup generated by the system of conservation laws (1.1). A function $u:[0, T] \mapsto \mathcal{D}$ is a viscosity solution of (1.1) if and only if $u(t)=S_{t} u(0)$ for all $t \in[0, T]$.

\section{Vanishing viscosity approximations}

It is natural to expect that the entropy weak solutions of the hyperbolic system (1.1) actually coincide with the limits of solutions to the parabolic system

$$
u_{t}^{\varepsilon}+f\left(u^{\varepsilon}\right)_{x}=\varepsilon u_{x x}^{\varepsilon}
$$

letting the viscosity coefficient $\varepsilon \rightarrow 0$. For smooth solutions, this convergence result is easy to show. However, one should keep in mind that a weak solution of the hyperbolic system (1.1) in general is only a function with bounded variation, possibly with a countable number of discontinuities. In this case, as the smooth functions $u^{\varepsilon}$ approach the discontinuous solution $u$, near points of jump their gradients $u_{x}^{\varepsilon}$ must tend to infinity (fig. 12), while their second derivatives $u_{x x}^{\varepsilon}$ become even more singular. Therefore, the convergence $u^{\varepsilon} \rightarrow u$ of the vanishing viscosity limit is a highly nontrivial matter.

In earlier literature, results in this direction relied on three different approaches:

1 - Comparison principles for parabolic equations. For a scalar conservation law, the existence, uniqueness and global stability of vanishing viscosity solutions 


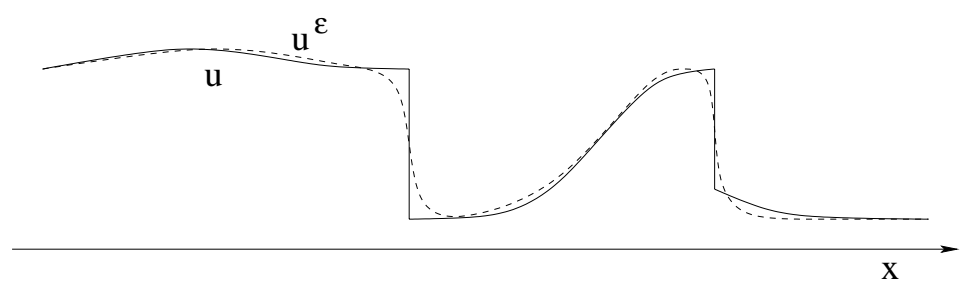

figure 12

was first established by Oleinik [O] in one space dimension. The famous paper by Kruzhkov $[\mathrm{K}]$ covers the more general class of $\mathbf{L}^{\infty}$ solutions and is also valid in several space dimensions.

2 - Singular perturbations. This technique was developed by Goodman and Xin [GX], and covers the case where the limit solution $u$ is piecewise smooth, with a finite number of non-interacting, entropy admissible shocks. See also [Y] and [Ro], for further results in this direction.

3 - Compensated compactness. With this approach, introduced by Tartar and DiPerna [DP2], one first considers a weakly convergent subsequence $u^{\varepsilon} \rightarrow$ $u$. For a class of $2 \times 2$ systems, one can show that this weak limit $u$ actually provides a distributional solution to the nonlinear system (1.1). The proof relies on a compensated compactness argument, based on the representation of the weak limit in terms of Young measures, which must reduce to a Dirac mass due to the presence of a large family of entropies.

In view of recent results on the well-posedness of the hyperbolic Cauchy problem within the space of BV functions, it is natural to seek uniform BV bounds also on the viscous approximations $u^{\varepsilon}$ in (1.3). This is indeed the main goal accomplished in [BB]. As soon as these BV bounds are established, the existence of a vanishing viscosity limit follows by a standard compactness argument. The uniqueness of the limit can then be deduced from the uniqueness theorem in [BG]. By further analysis, one can also prove the continuous dependence on the initial data for the viscous approximations $u^{\varepsilon}$, in the $\mathbf{L}^{1}$ norm. These results are valid, more generally, for hyperbolic systems not necessarily in conservation form. In this case, however, the unique limits of vanishing viscosity approximations cannot be interpreted as weak solutions in a distributional sense. Their characterization thus requires more care. The main results in $[\mathrm{BB}]$ can be stated as follows.

Theorem 5. Consider the Cauchy problem for the hyperbolic system with viscosity

$$
u_{t}^{\varepsilon}+A\left(u^{\varepsilon}\right) u_{x}^{\varepsilon}=\varepsilon u_{x x}^{\varepsilon} \quad u^{\varepsilon}(0, x)=\bar{u}(x) .
$$

Assume that the matrices $A(u)$ are strictly hyperbolic, smoothly depending on $u$ in a neighborhood of the origin. Then there exist constants $C, L, L^{\prime}$ and $\delta>0$ such that the following holds. If

$$
\|\bar{u}\|_{\mathbf{L}^{\infty}}<\delta, \quad \text { Tot.Var. }\{\bar{u}\}<\delta,
$$


then for every $\varepsilon>0$ the Cauchy problem (6.2) has a unique solution $u^{\varepsilon}$, defined for all $t \geq 0$. Adopting a semigroup notation, this will be written as $t \mapsto u^{\varepsilon}(t, \cdot) \doteq S_{t}^{\varepsilon} \bar{u}$. In addition, one has:

BV bounds :

$$
\text { Tot.Var. }\left\{S_{t}^{\varepsilon} \bar{u}\right\} \leq C \text { Tot.Var. }\{\bar{u}\} \text {. }
$$

$\mathbf{L}^{1}$ stability :

$$
\begin{array}{r}
\left\|S_{t}^{\varepsilon} \bar{u}-S_{t}^{\varepsilon} \bar{v}\right\|_{\mathbf{L}^{1}} \leq L\|\bar{u}-\bar{v}\|_{\mathbf{L}^{1}}, \\
\left\|S_{t}^{\varepsilon} \bar{u}-S_{s}^{\varepsilon} \bar{u}\right\|_{\mathbf{L}^{1}} \leq L^{\prime}(|t-s|+|\sqrt{\varepsilon t}-\sqrt{\varepsilon s}|) .
\end{array}
$$

Convergence: $A s \varepsilon \rightarrow 0+$, the solutions $u^{\varepsilon}$ converge to the trajectories of a semigroup $S$ such that

$$
\left\|S_{t} \bar{u}-S_{s} \bar{v}\right\|_{\mathbf{L}^{1}} \leq L\|\bar{u}-\bar{v}\|_{\mathbf{L}^{1}}+L^{\prime}|t-s| .
$$

These vanishing viscosity limits can be regarded as the unique vanishing viscosity solutions of the hyperbolic Cauchy problem

$$
u_{t}+A(u) u_{x}=0, \quad u(0, x)=\bar{u}(x) .
$$

In the conservative case $A(u)=D f(u)$, every vanishing viscosity solution is a weak solution of

$$
u_{t}+f(u)_{x}=0, \quad u(0, x)=\bar{u}(x),
$$

satisfying the Liu admissibility conditions [L1].

We outline here the main ideas in the proof of Theorem 5 .

1. The rescaling $t \mapsto t / \varepsilon, x \mapsto x / \varepsilon$ transforms the problem (6.2) into

$$
u_{t}+A(u) u_{x}=u_{x x} \quad u(0, x)=\bar{u}(\varepsilon x) .
$$

Clearly, the total variation of a solution $u$ is not affected by this rescaling. Notice however that the values of a solution $u^{\varepsilon}$ of $(6.2)$ on a time interval $[0, T]$ correspond to the value of a solution $u$ of $(6.9)$ on the much longer interval $[0, T / \varepsilon]$. To obtain BV estimates uniformly valid for all $\varepsilon>0$, we thus need to prove similar estimates for the problem

$$
u_{t}+A(u) u_{x}=u_{x x}
$$

uniformly valid for all $t \in[0, \infty[$ and all initial data $u(0, \cdot)$ with suitably small total variation.

2. From now on we thus consider the parabolic system (6.10). On an initial time interval $[0, T]$, the solution can be represented as

$$
u(t)=G(t) * u(0)-\int_{0}^{t} G(t-s) * A(u(s)) u_{x}(s) d s,
$$

in terms of convolutions with the standard heat kernel $G(t, x) \doteq(4 \pi t)^{-1 / 2} e^{-x^{2} / 4 t}$. Even if the initial data is discontinuous, the presence of viscosity has an immediate regularizing effect and the solution becomes smooth for all times $t>0$. The smoothing effect of the parabolic equation is summarized in fig. 13. Let

$$
\delta_{0} \doteq \text { Tot.Var. }\{\bar{u}\}
$$

be the total variation of the initial data. Then, on an initial interval $t \in] 0, T]$ with $T=\mathcal{O}(1) \cdot \delta_{0}^{-2}$ one can show that 


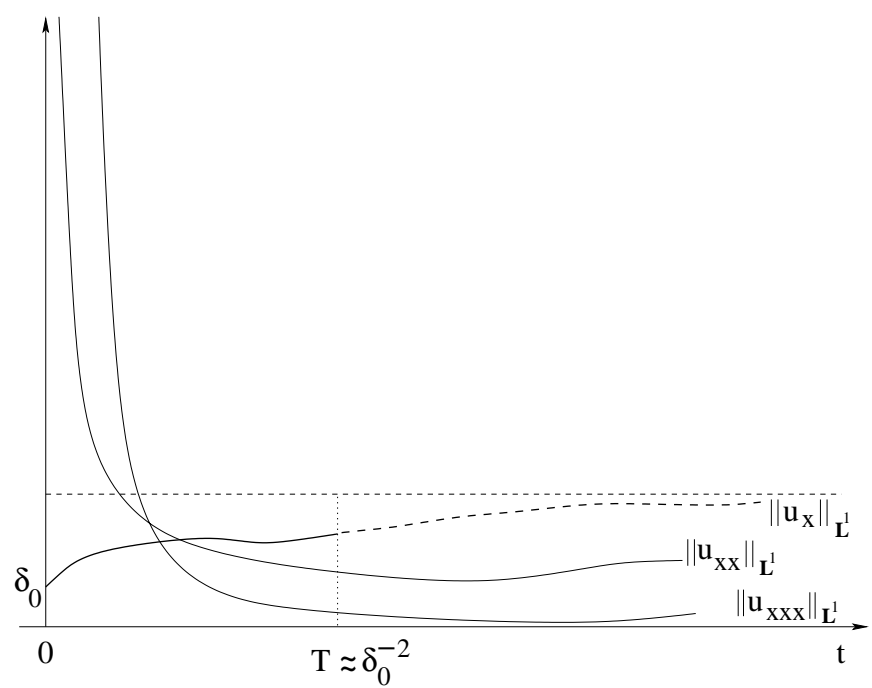

figure 13

- The total variation of $u(t, \cdot)$ remains bounded, i.e.

$$
\left\|u_{x}(t, \cdot)\right\|_{\mathbf{L}^{1}}=\mathcal{O}(1) \cdot \delta_{0}
$$

- The norms of all higher order derivatives decay at algebraic rates. In particular

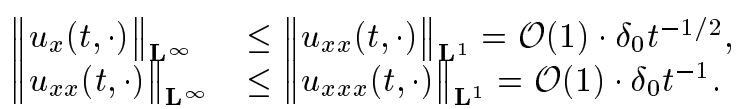

All these bounds can be derived by looking at integral representations of the form (6.11).

For $t \in[T, \infty[$, these parabolic type estimates yield only partial informations. What can be proved is the following: as long as the bound (6.12) on the total variation remains valid, also the norms of all higher derivatives remain small. Namely

$$
\left\|u_{x}(t, \cdot)\right\|_{\mathbf{L}^{\infty}} \leq\left\|u_{x x}(t, \cdot)\right\|_{\mathbf{L}^{1}}=\mathcal{O}(1) \cdot \delta_{0}^{2},
$$

3. The main part of the proof is to establish the uniform bound (6.12) on the total variation, for all times $t \in[0, \infty[$. By the previous step, we can assume that the bounds (6.14) on higher order derivatives already hold. As mentioned in the Introduction, the basic approach is as follows. At each point $(t, x)$ we decompose the gradient along a suitable basis of unit vectors $\tilde{r}_{i}$, say

$$
u_{x}=\sum v_{i} \tilde{r}_{i}
$$

Then we derive an evolution equation for these gradient components, and show that their $\mathbf{L}^{1}$ norm remains uniformly bounded.

Toward this decomposition, the first step is to select $n$ families of travelling wave profiles $U_{i}$, each depending on $n+2$ scalar parameters. This will allows us to solve the system (1.11)-(1.12) in unique way, depending continuously on the data $\left(u, u_{x}, u_{x x}\right)$. 


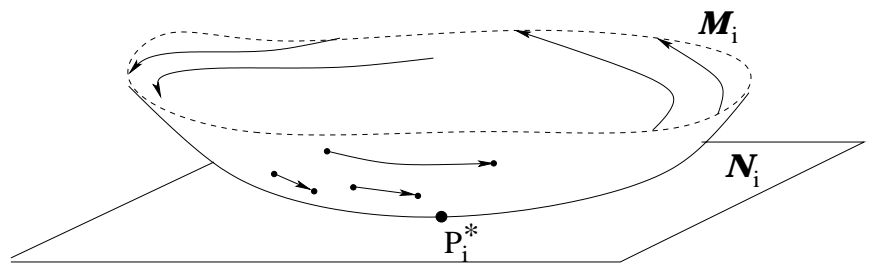

figure 14

4. A viscous travelling $i$-wave profile is a solution of (1.10), where the speed $\sigma$ is close to the $i$-th eigenvalue $\lambda_{i}$ of the hyperbolic matrix $A$. This second order O.D.E. can be rewritten as a first order system:

$$
\left\{\begin{array}{l}
\dot{u}=v \\
\dot{v}=(A(u)-\sigma) v \\
\dot{\sigma}=0
\end{array}\right.
$$

Linearizing at a point $P_{i}^{*}=\left(u^{*}, 0, \lambda_{i}\left(u^{*}\right)\right)$ we obtain the linear system with constant coefficients

$$
\left\{\begin{array}{l}
\dot{u}=v \\
\dot{v}=\left(A\left(u^{*}\right)-\lambda_{i}\left(u^{*}\right)\right) v, \\
\dot{\sigma}=0
\end{array}\right.
$$

This system admits a center subspace $\mathcal{N}_{i}$ of dimension $n+2$. By the center manifold theorem [V], there exists a manifold $\mathcal{M}_{i}$, tangent to $\mathcal{N}_{i}$ at the point $P_{i}^{*}$ (see fig. 14), locally invariant for the flow of the nonlinear system (6.16). Instead of looking at all viscous travelling profiles, we can now restrict ourselves to only those profiles corresponding to a trajectory on the center manifold $\mathcal{M}_{i}$. Since this manifold is $(n+2)$-dimensional, through each state $u \in \mathbb{R}^{n}$ there is a 2-parameter family of such profiles, say $U_{i}\left(u, v_{i}, \sigma_{i}\right)$. Here $v_{i}$ denotes the scalar amplitude of the wave, and $\sigma_{i}$ is the speed. Calling $\tilde{r}_{i}\left(u, v_{i}, \sigma_{i}\right)$ the unit vector parallel to $U_{i}^{\prime}$, this profile $U_{i}$ is characterized by the equations

$$
U_{i}^{\prime \prime}=\left(A\left(U_{i}\right)-\sigma_{i}\right) U_{i}^{\prime}, \quad U_{i}(0)=u, \quad U_{i}^{\prime}(0)=v_{i} \tilde{r}_{i}
$$

The manifold can then be defined as

$$
\mathcal{M}_{i}=\left\{\left(u, v, \sigma_{i}\right) \in \mathbb{R}^{n+n+1} ; v=v_{i} \tilde{r}_{i}\left(u, v_{i}, \sigma_{i}\right)\right\} .
$$

We can now try to solve the system (1.11)-(1.12) using not all travelling wave profiles in (1.10), but only those $U_{i}$ which correspond to a trajectory on the center manifold $\mathcal{M}_{i}$. Notice that in this case we get the correct number of parameters: in all, the $n$ profiles $U_{1}, \ldots, U_{n}$ through the point $u(x)$ depend on the $2 n$ scalar parameters $v_{1}, \sigma_{1}, \ldots, v_{n}, \sigma_{n}$. Exactly the right number to fit the data $\left(u_{x}(x), u_{x x}(x)\right) \in \mathbb{R}^{n+n}$.

To better understand how our decomposition works, consider first the special case where $u$ is precisely the profile of a viscous travelling wave of the $j$-th family. In this case, the decomposition should clearly contain one single component:

$$
u_{x}=v_{j} \tilde{r}_{j}\left(u, v_{j}, \sigma_{j}\right) .
$$


It is easy to guess what $v_{j}$ and $\sigma_{j}$ should be. Indeed, since by construction $\left|\tilde{r}_{j}\right|=1$, the quantity

$$
v_{j}= \pm\left|u_{x}\right|
$$

is the signed strength of the wave. We also notice that, for a travelling wave, the vectors $u_{x}$ and $u_{t}$ are always parallel. Indeed, $u_{t}=-\sigma_{j} u_{x}$, where $\sigma_{j}$ is the speed of the wave. We can thus write

$$
u_{t}=u_{x x}-A(u) u_{x}=\omega_{j} \tilde{r}_{j}\left(u, v_{j}, \sigma_{j}\right)
$$

for some scalar $\omega_{j}$. The speed of the wave is now obtained as $\sigma_{j}=-\omega_{j} / v_{j}$.

Motivated by the previous analysis, as a first attempt we define our decomposition in terms of the equations

$$
\left\{\begin{array}{l}
u_{x}=\sum_{i} v_{i} \tilde{r}_{i}\left(u, v_{i}, \sigma_{i}\right), \\
u_{t}=\sum_{i} \omega_{i} \tilde{r}_{i}\left(u, v_{i}, \sigma_{i}\right),
\end{array} \quad \sigma_{i}=-\frac{\omega_{i}}{v_{i}} .\right.
$$

The trouble with (6.20) is that the vectors $\tilde{r}_{i}$ are defined only for speeds $\sigma_{i}$ close to the $i$-th characteristic speed $\lambda_{i}^{*} \doteq \lambda_{i}\left(u^{*}\right)$. However, when $u_{x} \approx 0$ one has $v_{i} \approx 0$ and the ratio $\omega_{i} / v_{i}$ may become arbitrarily large. To overcome this problem, we introduce a smooth cutoff function $\theta$ such that

$$
\theta(s)=\left\{\begin{array}{lll}
s & \text { if } & |s| \leq \delta_{1} \\
0 & \text { if } & |s| \geq 3 \delta_{1}
\end{array}\right.
$$

for some small constant $\delta_{1}>0$. We now rewrite (6.20) in terms of the new variable $w_{i}$, related to $\omega_{i}$ by $\omega_{i} \doteq w_{i}-\lambda_{i}^{*} v_{i}$. We require that $\sigma_{i}$ coincides with $-\omega_{i} / v_{i}$ only when this ratio is sufficiently close to $\lambda_{i}^{*} \doteq \lambda_{i}\left(u^{*}\right)$. Our basic equations thus take the form

$$
\left\{\begin{array}{l}
u_{x}=\sum_{i} v_{i} \tilde{r}_{i}\left(u, v_{i}, \sigma_{i}\right) \\
u_{t}=\sum_{i}\left(w_{i}-\lambda_{i}^{*} v_{i}\right) \tilde{r}_{i}\left(u, v_{i}, \sigma_{i}\right)
\end{array}\right.
$$

where

$$
u_{t}=u_{x x}-A(u) u_{x}, \quad \sigma_{i}=\lambda_{i}^{*}-\theta\left(\frac{w_{i}}{v_{i}}\right) .
$$

The system (6.22)-(6.23) can be written in the more compact form

$$
\left(\begin{array}{l}
u_{x} \\
u_{t}
\end{array}\right)=\Lambda(u, v, w) \text {. }
$$

Using the implicit function theorem one can prove that, for each given state $u \approx$ $u^{*}$ and $u_{x}, u_{t}$ sufficiently small, there exists unique $v=\left(v_{1}, \ldots, v_{n}\right)$ and $w=$ $\left(w_{1}, \ldots, w_{n}\right)$ such that $(6.22)-(6.23)$ hold. Furthermore, the map $\left(u, u_{x}, u_{t}\right) \mapsto$ $(v, w)$ is $\mathcal{C}^{1}$ with Lipschitz continuous first derivative. This achieves the desired decomposition of the gradient $u_{x}$.

We observe that above equations are closely related to (1.12). Indeed, recalling (6.18), from the first equation in (6.22) it follows

$$
u_{x}(x)=\sum_{i} U_{i}^{\prime}(x) .
$$

If $\sigma_{i}=\lambda_{i}^{*}-w_{i} / v_{i}$ for all $i=1, \ldots, n$, i.e. if none of the cutoff functions is active, then

$$
\begin{aligned}
u_{x x}(x) & =u_{t}+A(u) u_{x}=\sum_{i}\left(w_{i}-\lambda_{i}^{*} v_{i}\right) \tilde{r}_{i}+A(u) \sum_{i} v_{i} \tilde{r}_{i} \\
& =\sum_{i}\left(A(u)-\sigma_{i}\right) v_{i} \tilde{r}_{i}=\sum_{i} U_{i}^{\prime \prime}(x) .
\end{aligned}
$$

In this case, both of the equalities in (1.12) hold. Notice however that the second equality in (1.12) may fail if $\left|w_{i} / v_{i}\right|>\delta_{1}$ for some $i$, so that $\theta\left(w_{i} / v_{i}\right) \neq w_{i} / v_{i}$. 
Remark 1. For each $i=1, \ldots, n$, in addition to the eigenvalue $\lambda_{i}(u)$ of the matrix $A(u)$, one can define the viscous eigenvalue $\tilde{\lambda}_{i}\left(u, v_{i}, \sigma_{i}\right)$ by setting

$$
\tilde{\lambda}_{i} \doteq\left\langle\tilde{r}_{i}, A(u) \tilde{r}_{i}\right\rangle .
$$

Instead of the standard relation

$$
\left(A(u)-\lambda_{i}\right) r_{i}=0
$$

a direct computation shows that these viscous eigenvectors satisfy

$$
\left(A(u)-\tilde{\lambda}_{i}\right) \tilde{r}_{i}=v_{i}\left(\tilde{r}_{i, u} \tilde{r}_{i}+\tilde{r}_{i, v}\left(\tilde{\lambda}_{i}-\sigma_{i}\right)\right) .
$$

Here $\tilde{r}_{i, u}=\partial \tilde{r}_{i} / \partial u$ and $\tilde{r}_{i, v}=\partial \tilde{r}_{i} / \partial v_{i}$ denote partial derivatives. In the special case $v_{i}=0$, an easy consequence of (6.26) is the identity

$$
\tilde{r}_{i}\left(u, 0, \sigma_{i}\right)=r_{i}(u) \text {. }
$$

The presence of non-zero terms on the right hand side of (6.26) is of fundamental importance. Indeed, in the evolution equation for the component $v_{i}$, these terms achieve a crucial cancellation with other terms that would otherwise not be integrable.

5. We now consider a smooth solution $u=u(t, x)$ of the parabolic system (6.10). By the previous step, we can define the scalar functions $v_{i}=v_{i}(t, x), w_{i}=w_{i}(t, x)$, according to the decomposition (6.22)-(6.23). The equations governing the evolution of these $2 n$ components can be written in the form

$$
\begin{cases}v_{i, t}+\left(\tilde{\lambda}_{i} v_{i}\right)_{x}-v_{i, x x} & =\phi_{i} \\ w_{i, t}+\left(\tilde{\lambda}_{i} w_{i}\right)_{x}-w_{i, x x} & =\psi_{i}\end{cases}
$$

We recall that $\tilde{\lambda}_{i}$ is the speed defined at (6.25). The source terms $\phi_{i}, \psi_{i}$ can be computed by inserting (6.24) in the evolution equation

$$
\left(\begin{array}{l}
u_{x} \\
u_{t}
\end{array}\right)_{t}+\left(\left[\begin{array}{cc}
A(u) & 0 \\
0 & A(u)
\end{array}\right]\left(\begin{array}{l}
u_{x} \\
u_{t}
\end{array}\right)\right)_{x}-\left(\begin{array}{l}
u_{x} \\
u_{t}
\end{array}\right) x_{x}=\left(\begin{array}{c}
0 \\
\left(D A(u) \cdot u_{x}\right) u_{t}-\left(D A(u) \cdot u_{t}\right) u_{x}
\end{array}\right)
$$

and differentiating various times w.r.t. $t, x$, using the fundamental relation (6.26). For all details we refer to [BB]. Rather than the exact form of the source terms, what matters here is their magnitude. This can be estimated as follows.

Lemma 1. The source terms in (6.27) satisfy the bounds

$$
\begin{aligned}
\phi_{i}, \psi_{i}= & \mathcal{O}(1) \cdot \sum_{j}\left(\left|v_{j, x}\right|+\left|w_{j, x}\right|\right) \cdot\left|w_{j}-\theta_{j} v_{j}\right| \quad \text { (cutoff error) } \\
& +\mathcal{O}(1) \cdot \sum_{j \neq k}\left(\left|v_{j} v_{k}\right|+\left|v_{j, x} v_{k}\right|+\left|v_{j} w_{k}\right|+\left|v_{j, x} w_{k}\right|+\left|v_{j} w_{k, x}\right|+\left|w_{j} w_{k}\right|\right) \\
& \quad \text { (interaction of waves of different families) } \\
+ & \mathcal{O}(1) \cdot \sum_{j}\left|v_{j, x} w_{j}-v_{j} w_{j, x}\right|+\mathcal{O}(1) \cdot \sum_{j}\left|v_{j}\left(\frac{w_{j}}{v_{j}}\right)_{x}\right|^{2} \cdot \chi_{\left\{\left|w_{j} / v_{j}\right|<3 \delta_{1}\right\}}
\end{aligned}
$$

Here $\delta_{1}$ is the constant in the cutoff function at (6.21). A rigorous proof of Lemma 1 requires lengthy calculations. Here we shall only provide an intuitive explanation of how the various terms arise. Consider first the special case where $u$ is precisely one of the travelling wave profiles on the center manifold (fig. 15a), say $u(t, x)=U_{j}\left(x-\sigma_{j} t\right)$. In this case one has

$$
u_{x}=v_{j} \tilde{r}_{j}, \quad u_{t}=\left(w_{j}-\lambda_{j}^{*} v_{j}\right) \tilde{r}_{j}, \quad v_{i}=w_{i}=0 \quad \text { for } i \neq j,
$$




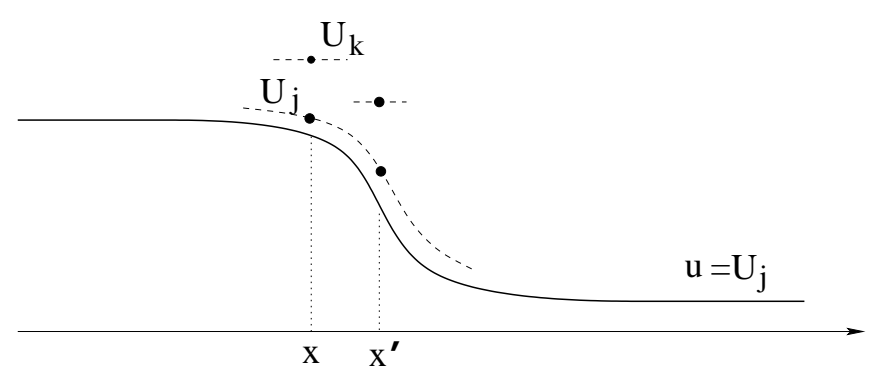

figure $15 \mathrm{a}$

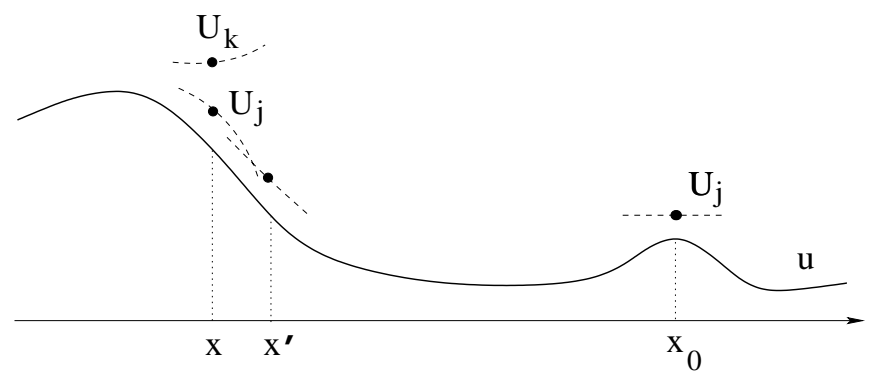

figure $15 \mathrm{~b}$

and all source terms $\phi_{i}, \psi_{i}$ vanish identically.

Next, consider the case of a general solution $u=u(t, x)$ of $(6.10)$. The sources on the right hand sides of (6.27) arise for three different reasons (fig. 15b).

(i) The ratio $\left|w_{j} / v_{j}\right|$ is large and hence the cutoff function $\theta_{j} \doteq \theta\left(w_{j} / v_{j}\right)$ is active. Typically, this will happen near a point $x_{0}$ where $u_{x}=0$ but $u_{t}=u_{x x} \neq 0$. In this case the second identity in (1.12) fails because of a "wrong" choice of the speed: $\sigma_{j} \neq \lambda_{j}^{*}-\left(w_{j} / v_{j}\right)$.

(ii) Waves of two different families $j \neq k$ are present at a given point $x$. These will produce quadratic source terms, due to transversal interactions.

(iii) Since the decomposition (6.22) is defined pointwise, it may well happen that the travelling $j$-wave profile $U_{j}$ at a point $x$ is not the same as the profile $U_{j}$ at a nearby point $x^{\prime}$. Indeed, these two travelling waves may have slightly different speeds. It is the rate of change in this speed: $\sigma_{j, x}=-\left(w_{j} / v_{j}\right)_{x}$, that determines the infinitesimal interaction between nearby waves of the same family. A detailed analysis shows that the corresponding source terms can only be linear or quadratic w.r.t. $\sigma_{j, x}$, with the square of the strength of the wave always appearing as a factor. These terms can thus be estimated as $\mathcal{O}(1) \cdot v_{j}^{2} \sigma_{j, x}+\mathcal{O}(1) \cdot v_{j}^{2} \sigma_{j, x}^{2}$.

To complete the proof of the BV bounds, it remains to show that all terms on the right hand side of (6.28) are integrable over the domain $\{t>T, x \in \mathbb{R}\}$. For this purpose, we shall introduce four types of Lyapunov functionals, called energy, transversal interaction, curve length and area functional, monotonically decreasing 


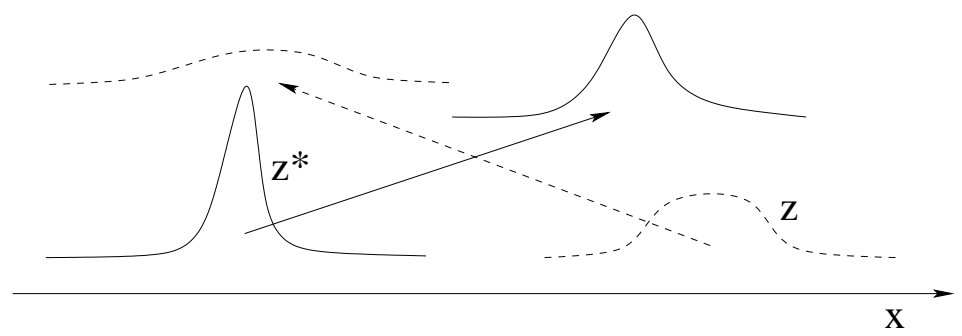

figure 16

along solutions. Each of the terms that we need to estimate will be dominated by the rate of decrease of one of these functionals.

\section{Lyapunov functionals}

We begin by describing a functional which controls the amount of interaction between viscous waves of different families.

Consider two independent, scalar diffusion equations with strictly different drifts (fig. 16):

$$
\begin{cases}z_{t}+[\lambda(t, x) z]_{x}-z_{x x} & =0 \\ z_{t}^{*}+\left[\lambda^{*}(t, x) z^{*}\right]_{x}-z_{x x}^{*} & =0\end{cases}
$$

assuming that

$$
\inf _{t, x} \lambda^{*}(t, x)-\sup _{t, x} \lambda(t, x) \geq c>0 .
$$

We can think $z$ as the density of waves with a slow speed $\lambda$ and $z^{*}$ as the density of waves with a fast speed $\lambda^{*}$. We seek an a priori bound on the total amount of interaction

$$
\int_{0}^{\infty} \int_{\mathbb{R}}|z(t, x)| \cdot\left|z^{*}(t, x)\right| d x d t
$$

This can be achieved by means of a transversal interaction potential, defined by

$$
\begin{gathered}
Q\left(z, z^{*}\right) \doteq \frac{1}{c} \iint_{\mathbb{R}^{2}} K\left(x_{2}-x_{1}\right)\left|z\left(x_{1}\right)\right|\left|z^{*}\left(x_{2}\right)\right| d x_{1} d x_{2}, \\
K(y) \doteq\left\{\begin{array}{lll}
e^{-c y / 2} & \text { if } & \mathrm{y}_{i} 0, \\
1 & \text { if } & y \leq 0 .
\end{array}\right.
\end{gathered}
$$

Notice that, to the product of a slow wave located at $x_{1}$ and a fast wave at $x_{2}$, in (7.2) we assign weight $K\left(x_{2}-x_{1}\right)=1$ if the two waves are approaching (i.e. if $x_{1}>x_{2}$ ), and an exponentially small weight if they are moving away from each other (i.e. if $x_{1}<x_{2}$ ). It turns out that this functional is monotonically decreasing along every couple of solutions $z, z^{*}$. Moreover, the rate of decrease controls the 


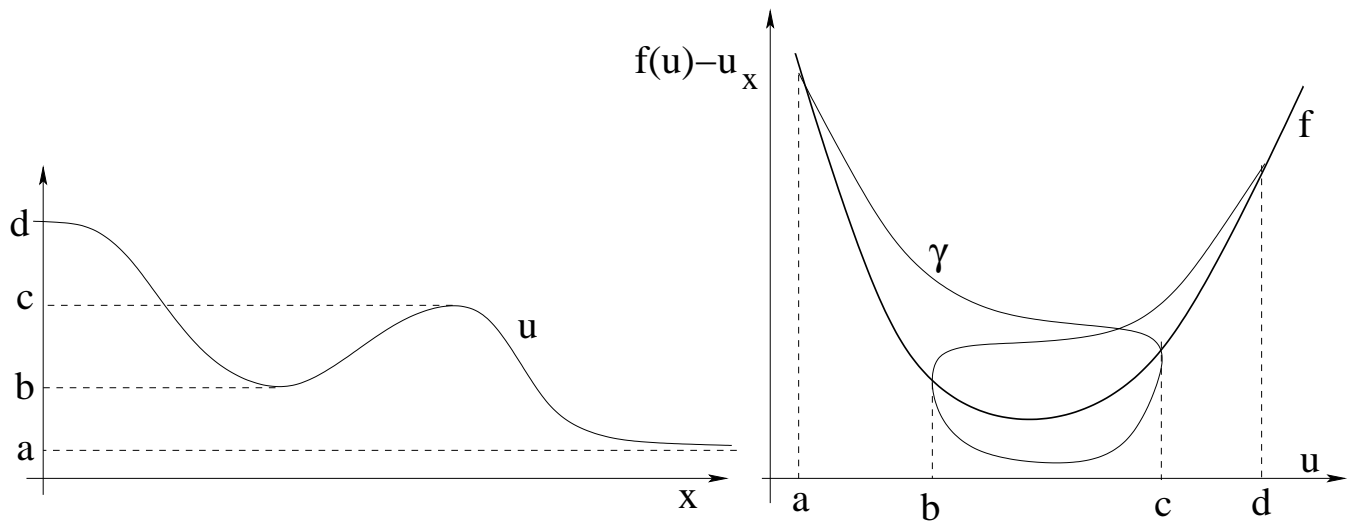

figure 17

integrand in (7.1). Namely

$$
\begin{gathered}
\int_{0}^{\infty} \int_{\mathbb{R}}|z(t, x)|\left|z^{*}(t, x)\right| d x d t \leq-\int_{0}^{\infty}\left[\frac{d}{d t} Q\left(z(t), z^{*}(t)\right)\right] d t \\
\leq Q\left(z(0), z^{*}(0)\right) \leq \frac{1}{c} \int_{\mathbb{R}}|z(0, x)| d x \cdot \int_{\mathbb{R}}\left|z^{*}(0, x)\right| d x .
\end{gathered}
$$

By introducing Lyapunov functionals of this type, we can bound the integrals of all terms of the form

$$
\left|v_{j} v_{k}\right|\left|v_{j, x} v_{k}\right|,\left|v_{j} w_{k}\right|,\left|v_{j, x} w_{k}\right|,\left|w_{j} w_{k}\right|
$$

with $j \neq k$, due to the interaction of waves of different families.

Next, we seek functionals which control the interactions between waves of the same family. Toward this goal, we first consider Lyapunov functionals which are decreasing along every solution of the scalar viscous conservation law

$$
u_{t}+f(u)_{x}=u_{x x}
$$

Given a scalar function $x \mapsto u(x)$, define the curve (fig. 17)

$$
\gamma \doteq\left(\begin{array}{c}
u \\
f(u)-u_{x}
\end{array}\right)=\left(\begin{array}{c}
\text { conserved quantity } \\
\text { flux }
\end{array}\right)
$$

In connection with a solution $u=u(t, x)$ of $(7.4)$, the curve $\gamma$ evolves according to

$$
\gamma_{t}+f^{\prime}(u) \gamma_{x}=\gamma_{x x}
$$

Notice that the vector $f^{\prime}(u) \gamma_{x}$ is parallel to $\gamma$, hence the presence of this term in (7.6) only amounts to a reparametrization of the curve, and does not affect its shape. The curve thus evolves in the direction of curvature. An obvious Lyapunov functional is thus the length of the curve. In terms of the variables

$$
\gamma_{x}=\left(\begin{array}{c}
v \\
w
\end{array}\right) \doteq\left(\begin{array}{c}
u_{x} \\
-u_{t}
\end{array}\right)
$$




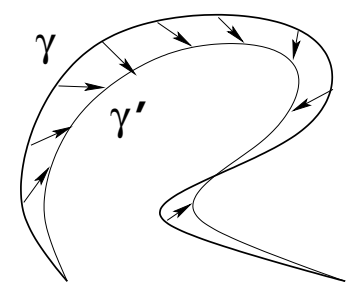

figure $18 \mathrm{a}$

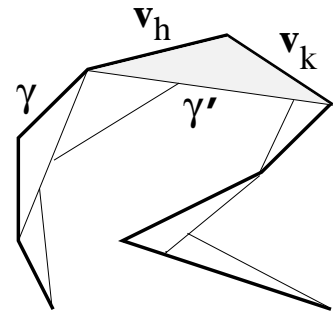

figure $18 \mathrm{~b}$

this length is given by

$$
L(\gamma) \doteq \int\left|\gamma_{x}\right| d x=\int \sqrt{v^{2}+w^{2}} d x
$$

We can estimate the rate of decrease of the length as

$$
-\frac{d}{d t} L(\gamma(t))=\int_{\mathbb{R}} \frac{|v|\left[(w / v)_{x}\right]^{2}}{\left(1+(w / v)^{2}\right)^{3 / 2}} d x \geq \frac{1}{\left(1+\delta^{2}\right)^{3 / 2}} \int_{|w / v| \leq \delta}|v|\left[(w / v)_{x}\right]^{2} d x,
$$

for any given constant $\delta>0$. Therefore

$$
\int_{0}^{\infty} \int_{|w / v| \leq \delta_{1}}|v|\left[(w / v)_{x}\right]^{2} d x d t=\mathcal{O}(1) \cdot \int_{0}^{\infty}\left|\frac{d}{d t} L(\gamma(t))\right| d t=\mathcal{O}(1) \cdot L(\gamma(0))
$$

This argument allows us to estimate all source terms in (6.28) of the form

$$
\mathcal{O}(1) \cdot\left|v_{j}\left(\frac{w_{j}}{v_{j}}\right)_{x}\right|^{2} \cdot \chi_{\left\{\left|w_{j} / v_{j}\right|<3 \delta_{1}\right\}} \cdot
$$

In connection with the same curve $\gamma$ in (7.5), we now introduce a second functional, defined in terms of a wedge product.

$$
Q(\gamma) \doteq \frac{1}{2} \iint_{x<x^{\prime}}\left|\gamma_{x}(x) \wedge \gamma_{x}\left(x^{\prime}\right)\right| d x d x^{\prime}
$$

For any curve that moves in the plane in the direction of curvature (fig. 18a), this functional is monotone decreasing and its decrease controls the area swept by the curve: $|d A| \leq-d Q$. An intuitive proof of this fact can be obtained by looking at polygonal approximations. In the special case (fig. 18b) where $\gamma$ is a polygonal with edges $\mathbf{v}_{1}, \ldots, \mathbf{v}_{m}$, the functional in (7.9) reduces to

$$
Q(\gamma)=\frac{1}{2} \sum_{i<j}\left|\mathbf{v}_{i} \wedge \mathbf{v}_{j}\right|
$$

Consider now a second polygonal $\gamma^{\prime}$, obtained from $\gamma$ by replacing two consecutive edges $\mathbf{v}_{h}, \mathbf{v}_{k}$ by a single one. The area of the triangle between $\gamma$ and $\gamma^{\prime}$ is then

$$
\text { Area }=\frac{1}{2}\left|\mathbf{v}_{h} \wedge \mathbf{v}_{k}\right| \leq Q(\gamma)-Q\left(\gamma^{\prime}\right)
$$

Observing that every continuous curve moving in the direction of curvature can be approximated by a suitable sequence of polygonals, the relation $|d A| \leq-d Q$ is obtained in the limit. 
Using (7.6)-(7.7) we now compute

$$
-\frac{d Q}{d t} \geq\left|\frac{d A}{d t}\right|=\int\left|\gamma_{t} \wedge \gamma_{x}\right| d x=\int\left|\gamma_{x x} \wedge \gamma_{x}\right| d x=\int\left|v_{x} w-v w_{x}\right| d x .
$$

Therefore, integrating w.r.t. time one finds

$$
\int_{0}^{\infty} \int\left|v_{x} w-v w_{x}\right| d x d t \leq \int_{0}^{\infty}\left|\frac{d}{d t} Q(\gamma(t))\right| d t \leq Q(\gamma(0)) .
$$

This argument allows us to estimate all source terms in (6.28) of the form

$$
\mathcal{O}(1) \cdot\left|v_{j, x} w_{j}-v_{j} w_{j, x}\right|
$$

Remark 2. Defining the speed of a scalar viscous wave as $\sigma(x) \doteq-u_{t}(x) / u_{x}(x)$, the area functional $Q(\gamma)$ in (7.9) can be written as

$$
\begin{aligned}
Q(\gamma) & =\frac{1}{2} \iint_{x<y}\left|u_{x}(x) u_{t}(y)-u_{t}(x) u_{x}(y)\right| d x d y \\
& =\frac{1}{2} \iint_{x<y}\left|u_{x}(x) d x\right| \cdot\left|u_{x}(y) d y\right| \cdot|\sigma(x)-\sigma(y)| \\
& =\frac{1}{2} \iint_{x<y}[\text { wave at } x] \times[\text { wave at } y] \times[\text { difference in speeds] } .
\end{aligned}
$$

It now becomes clear that the area functional can be regarded as an interaction potential between waves of the same family. Indeed, it closely resembles the functional used by T. P. Liu in [L5].

Remark 3. In the case where $u$ is precisely a viscous travelling wave, the curve $\gamma$ reduces to a segment. Assume now that the flux $f$ is genuinely nonlinear, say with $f^{\prime \prime} \geq c>0$. Consider a solution $u$ which initially consists of two viscous travelling waves, far apart from each other (fig. 19). To fix the ideas, let the first wave join a left state $a$ with a middle state $b$, and the second wave join the middle state $b$ with a right state $c$, with $a>b>c$. The strength of the two waves can be measured as $s=a-b, s^{\prime}=b-c$. The corresponding curve $\gamma$ is approximately given by two segments, joining the points $P \doteq(a, f(a)), Q \doteq(b, f(b)), P^{\prime} \doteq(c, f(c))$. After a long time $\tau \gg 0$, the two shocks will interact, merging into one single viscous shock. The curve $\gamma(\tau)$ thus reduces to one single segment, joining $P$ with $P^{\prime}$. The area swept by the curve is approximately the area of the triangle $P Q P^{\prime}$. The assumption of genuine nonlinearity implies

$$
\text { area swept }=\mathcal{O}(1) \cdot\left|s s^{\prime}\right|\left(|s|+\left|s^{\prime}\right|\right) .
$$

In this case, the decrease in the area functional is of cubic order w.r.t. the strengths $s, s^{\prime}$ of the interacting waves. This is indeed the correct order of magnitude needed to control the strength of new waves generated by the interaction in the genuinely nonlinear case. It is remarkable that this area functional gives the correct order of magnitude of waves generated by an interaction also for a general flux function $f$, not necessarily convex.

Finally, we describe two functionals which can be used to control the source terms in (6.28) due to the cutoff errors.

Recalling the constant $\delta_{1}$ in (6.21), we define a second cutoff function $\eta$ such that

$$
\eta(s)=\left\{\begin{array}{lll}
0 & \text { if } & |s| \leq 3 \delta_{1} / 5 \\
1 & \text { if } & |s| \geq 4 \delta_{1} / 5
\end{array}\right.
$$



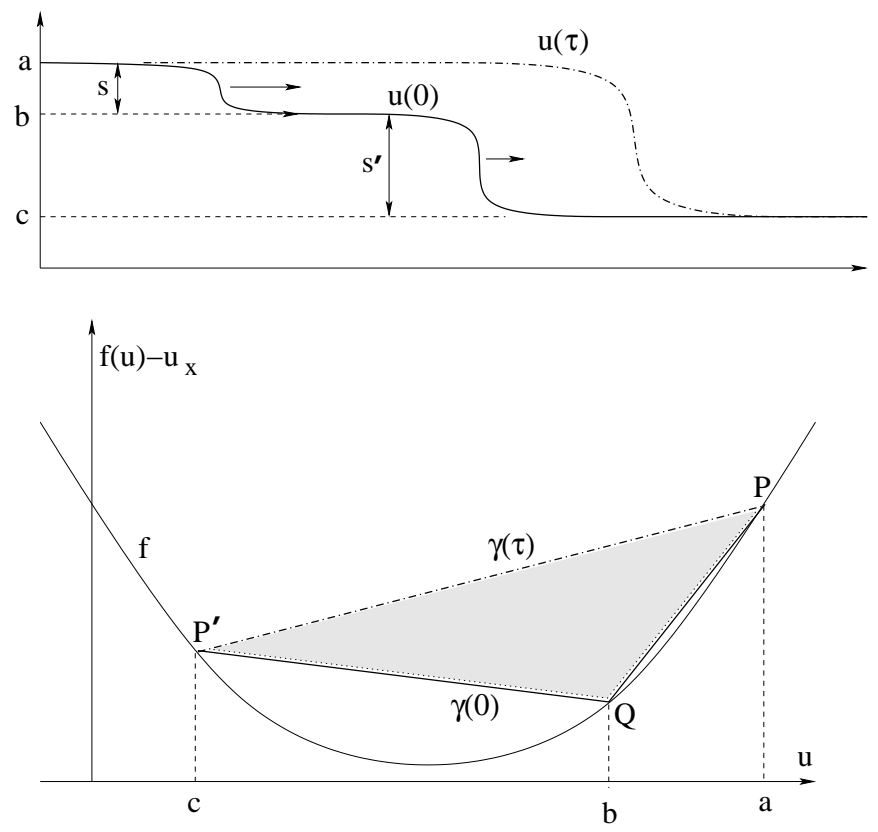

figure 19

In connection with a solution $\left(v_{i}, w_{i}\right)$ of $(6.27)$ we define $\eta_{i} \doteq \eta\left(w_{i} / v_{i}\right)$. The integral of the first terms on the right hand side of (6.28), due to the cutoff error, is then controlled by the decrease of the weighted energy functionals

$$
E_{i}(u) \doteq \int \eta_{i} \cdot v_{i, x}^{2} d x, \quad \widetilde{E}_{i}(u) \doteq \int \eta_{i} \cdot w_{i, x}^{2} d x
$$

For all details we always refer to [BB].

Remark 4. Intuitively, we expect that the cutoff function $\theta$ will be active when $\left|u_{x}\right|<<\left|u_{x x}\right|$. In these regions, the diffusion term $u_{x x}$ dominates over the convection term $A(u) u_{x}$ and the system (6.10) essentially reduces to the heat equation. It is thus natural to estimate the error due to cutoff in terms of an energy functional, similar to the one commonly used for the heat equation.

By means of the above functionals we obtain the $\mathbf{L}^{1}$ estimates on the source terms $\phi_{i}, \psi_{i}$ in (6.27), proving the uniform bounds on the total variation of a solution $u$ of (6.10). 


\section{Stability and convergence of viscous approximations}

To prove the uniform stability of all solutions of the parabolic system (6.10) having small total variation, we consider the linearized system describing the evolution of a first order variation. Inserting the formal expansion $u=u_{0}+\epsilon z+O\left(\epsilon^{2}\right)$ in (1.9), we obtain

$$
z_{t}+[D A(u) \cdot z] u_{x}+A(u) z_{x}=z_{x x} .
$$

A basic step in the proof is to establish the a priori bound

$$
\|z(t)\|_{\mathbf{L}^{1}} \leq L\|z(0)\|_{\mathbf{L}^{1}},
$$

for some constant $L$ and all $t \geq 0$ and every solution $z$ of the linearized system (8.1). By a standard homotopy argument, from (8.2) one easily deduces the Lipschitz continuity of the solution of (6.10) on the initial data. Namely, for every couple of solutions $u, \tilde{u}$ with small total variation one has

$$
\|u(t)-\tilde{u}(t)\|_{\mathbf{L}^{1}} \leq L\|u(0)-\tilde{u}(0)\|_{\mathbf{L}^{1}} .
$$

To see this, let any two solutions $u, \tilde{u}$ be given. We can connect them by a smooth path of solutions $u^{\theta}$, whose initial data satisfy

$$
u^{\theta}(0, x) \doteq \theta u(0, x)+(1-\theta) \tilde{u}(0, x), \quad \theta \in[0,1] .
$$

The distance $\|u(t, \cdot)-\tilde{u}(t, \cdot)\|_{\mathbf{L}^{1}}$ at any later time $t>0$ is clearly bounded by the length of the path $\theta \mapsto u^{\theta}(t)$. In turn, this can be computed by integrating the norm of a tangent vector. Calling $z^{\theta} \doteq d u^{\theta} / d \theta$, each vector $z^{\theta}$ is a solution of the corresponding equation (8.1), with $u$ replaced by $u^{\theta}$. Using (8.2) we thus obtain

$$
\begin{aligned}
& \|u(t \quad, \cdot)-\tilde{u}(t, \cdot)\|_{\mathbf{L}^{1}} \leq \int_{0}^{1}\left\|\frac{d}{d \theta} u^{\theta}(t)\right\|_{\mathbf{L}^{1}} d \theta=\int_{0}^{1}\left\|z^{\theta}(t)\right\|_{\mathbf{L}^{1}} d \theta \\
& \quad \leq L \int_{0}^{1}\left\|z^{\theta}(0)\right\|_{\mathbf{L}^{1}} d \theta=L\|u(0, \cdot)-\tilde{u}(0, \cdot)\|_{\mathbf{L}^{1}} \cdot
\end{aligned}
$$

By the simple rescaling of coordinates $t \mapsto \varepsilon t, x \mapsto \varepsilon x$, all of the above estimates remain valid for solutions $u^{\varepsilon}$ of the system (1.8). By a compactness argument, these $B V$ bounds imply the existence of a strong limit $u^{\varepsilon_{m}} \rightarrow u$ in $\mathbf{L}_{\text {loc }}^{1}$, at least for some subsequence $\varepsilon_{m} \rightarrow 0$. In the conservative case where $A=D f$, it is now easy to show that this limit $u$ provides a weak solution to the Cauchy problem (1.1).

To establish (8.2) one follows the same strategy as in the proof of the BV bounds. We decompose the vector $z$ as a sum of scalar components:

$$
z=\sum_{i} h_{i} \tilde{r}_{i}
$$

write an evolution equation for these components and prove that the source terms are integrable on the domain $\{t>0, x \in \mathbb{R}\}$.

Calling $\Upsilon \doteq z_{x}-A(u) z$, the decomposition takes the form

$$
\left\{\begin{array}{l}
z=\sum_{i} h_{i} \tilde{r}_{i}\left(u, v_{i}, \lambda_{i}^{*}-\theta\left(g_{i} / h_{i}\right)\right) \\
\Upsilon=\sum_{i}\left(g_{i}-\lambda_{i}^{*} h_{i}\right) \tilde{r}_{i}\left(u, v_{i}, \lambda_{i}^{*}-\theta\left(g_{i} / h_{i}\right)\right),
\end{array}\right.
$$

where $\theta$ is the cutoff function introduced at (6.21). These components $h_{i}, g_{i}$ satisfy the system of parabolic equations

$$
\begin{cases}h_{i, t}+\left(\tilde{\lambda}_{i} h_{i}\right)_{x}-h_{i, x x} & =\hat{\phi}_{i} \\ g_{i, t}+\left(\tilde{\lambda}_{i} g_{i}\right)_{x}-g_{i, x x} & =\hat{\psi}_{i}\end{cases}
$$


By similar arguments as in the previous sections, one obtains uniform bounds on all source terms $\hat{\phi}_{i}, \hat{\psi}_{i}$. This proves (8.2) and hence the stability of all solutions of (6.10) with small total variation.

Remark 5. The evolution equations (8.1) for a first order perturbation $z$ are linear. It thus seems natural to use a decomposition $\left(z, z_{x}\right) \mapsto(h, g)$ where the components $h_{i}, g_{i}$ are linear functions of $z, z_{x}$. This is the case if in (8.4) one chooses the same vectors $\tilde{r}_{i}=\tilde{r}_{i}\left(u, v_{i}, \sigma_{i}\right)$ as in (6.22), depending only on the reference solution $u$ and not on the perturbation $z$. Unfortunately, this linear decomposition does not seem to work, since it leads to a system of the form (8.6) with possibly non-integrable source terms. On the contrary, our decomposition (8.5) is positive homogeneous of degree one, but not linear. Indeed, the unit vectors $\tilde{r}_{i}$ depend on the reference solution $u$ through the amplitude $v_{i}$, but also on the perturbation $z$ implicitly through the speed $\sigma_{i}=\lambda_{i}^{*}-\theta\left(g_{i} / h_{i}\right)$.

For every initial data $u(0, \cdot)=\bar{u}$ with small total variation, the previous steps yield the existence of a unique global solution to the parabolic system (6.10), depending Lipschitz continuously on the initial data, in the $\mathbf{L}^{1}$ norm. Performing the rescaling $t \mapsto t / \varepsilon, x \mapsto x / \varepsilon$, we immediately obtain the same results for the Cauchy problem

$$
u_{t}^{\varepsilon}+A\left(u^{\varepsilon}\right) u_{x}^{\varepsilon}=\varepsilon u_{x x}^{\varepsilon}, \quad u^{\varepsilon}(0, x)=\bar{u}(x) .
$$

Adopting a semigroup notation, this solution will be written as $u^{\varepsilon}(t, \cdot)=S_{t}^{\varepsilon} \bar{u}$. Thanks to the uniform bounds on the total variation, a compactness argument yields the existence of a strong limit in $\mathbf{L}_{\text {loc }}^{1}$

$$
u=\lim _{\varepsilon_{m} \rightarrow 0} u^{\varepsilon_{m}},
$$

at least for some subsequence $\varepsilon_{m} \rightarrow 0$. Since the $u^{\varepsilon}$ depend continuously on the initial data, with a uniform Lipschitz constant, the same is true of $u(t, \cdot)=S_{t} \bar{u}$. In the conservative case where $A(u)=D f(u)$, it is not difficult to show that this limit $u$ actually provides a weak solution to the Cauchy problem (6.8).

The only remaining issue is to show that the limit in (8.6) is unique, i.e. it does not depend on the subsequence $\left\{\varepsilon_{m}\right\}$. In the standard conservative case, this fact can already be deduced from the uniqueness criterion in [BG]. In the general case, uniqueness is proved in two steps. First we show that, in the special case of a Riemann problem, the solution obtained as vanishing viscosity limit is unique and can be completely characterized. To conclude the proof, we then rely on the same general argument as in [B2]: if two Lipschitz semigroups $S, S^{\prime}$ provide the same solutions to all Riemann problems, then they must coincide.

\section{Concluding remarks}

It remains an open problem to establish the convergence of vanishing viscosity approximations of the form

$$
u_{t}+A(u) u_{x}=\varepsilon\left(B(u) u_{x}\right)_{x}
$$


for more general viscosity matrices $B$, as in [MP]. In the present paper we are exclusively concerned with the case where $B$ is the identity matrix. For systems which are not in conservative form, we expect that the limit of solutions of (9.1), as $\varepsilon \rightarrow 0$, will be heavily dependent on the choice of the matrix $B$. Studying the case where the viscosity matrix $B(u)$ is only semidefinite is particularly important: for applications to more realistic physical models and also toward the understanding of general relaxation approximations $[\mathrm{N}]$.

In the present survey we only considered initial data with small total variation. This is a convenient setting, adopted in much of the current literature, which guarantees the global existence of $B V$ solutions of (1.1) and captures the main features of the problem. In particular, a single solution can exhibit a rich structure, including shock formation, decay of genuinely nonlinear rarefaction waves, interaction and cancellation phenomena. On the other hand, from the point of view of dynamical systems, the global flow generated by a hyperbolic systems looks rather trivial: for large times, all small BV solutions asymptotically converge to a limit described by the solution of a Riemann problem. In particular, we are not yet in a position to add a large source term on the right hand side of (1.1) and study the resulting flow from a topological point of view, say determining periodic or heteroclinic orbits. The reason is, quite simply, that this rich dynamics can only be observed in solutions with large data. But in this case, unfortunately, no global existence theorem is yet known, except for very special systems.

A recent example of Jenssen $[\mathrm{J}]$ shows that, for initial data with large total variation, the $\mathbf{L}^{\infty}$ norm of the solution can blow up in finite time. In this more general setting, one can only expect that the existence and uniqueness of weak solutions, together with the convergence of vanishing viscosity approximations, will hold locally in time as long as the total variation remains bounded. For the hyperbolic system (1.1), results on the local existence and stability of solutions with large $B V$ data can be found in [Sc] and [BC2].

We remark that all of the blow up examples given so far arise from hyperbolic systems that do not admit any strictly convex entropy. This allows one to hope that, for physical systems, finite time blow up of solutions should not occur. At present, the role of entropies in preventing this finite time blow-up (as well as other pathological behavior) remains one of the major open problems in the onedimensional theory of hyerbolic conservation laws.

\section{References}

[1] P. Baiti and H. K. Jenssen, On the front tracking algorithm, J. Math. Anal. Appl. 217 (1998), 395-404.

[2] S. Bianchini and A. Bressan, Vanishing viscosity solutions to nonlinear hyperbolic systems, Annals of Mathematics, to appear.

[3] A. Bressan, Global solutions to systems of conservation laws by wave-front tracking, J. Math. Anal. Appl. 170 (1992), 414-432.

[4] A. Bressan, The unique limit of the Glimm scheme, Arch. Rational Mech. Anal. 130 (1995), 205-230. 
[5] A. Bressan, Hyperbolic Systems of Conservation Laws. The One Dimensional Cauchy Problem. Oxford University Press, 2000.

[6] A. Bressan and R. M. Colombo, The semigroup generated by $2 \times 2$ conservation laws, Arch. Rational Mech. Anal. 133 (1995), 1-75.

[7] A. Bressan and R. M. Colombo, Unique solutions of $2 \times 2$ conservation laws with large data, Indiana Univ. Math. J. 44 (1995), 677-725.

[8] A. Bressan, G. Crasta and B. Piccoli, Well posedness of the Cauchy problem for $n \times n$ conservation laws, Amer. Math. Soc. Memoir 694 (2000).

[9] A. Bressan and P. Goatin, Oleinik type estimates and uniqueness for $n \times n$ conservation laws, J. Diff. Equat. 156 (1999), 26-49.

[10] A. Bressan and P. LeFloch, Uniqueness of weak solutions to systems of conservation laws, Arch. Rat. Mech. Anal. 140 (1997), 301-317.

[11] A. Bressan and M. Lewicka, A uniqueness condition for hyperbolic systems of conservation laws, Discr. Cont. Dynam. Syst. 6 (2000), 673-682.

[12] A. Bressan, T. P. Liu and T. Yang, $L^{1}$ stability estimates for $n \times n$ conservation laws, Arch. Rational Mech. Anal. 149 (1999), 1-22.

[13] A. Bressan and A. Marson, Error bounds for a deterministic version of the Glimm scheme, Arch. Rational Mech. Anal. 142 (1998), 155-176.

[14] M. Crandall, The semigroup approach to first-order quasilinear equations in several space variables, Israel J. Math. 12 (1972), 108-132.

[15] C. Dafermos, Polygonal approximations of solutions of the initial value problem for a conservation law, J. Math. Anal. Appl. 38 (1972), 33-41.

[16] C. Dafermos, Hyperbolic Conservation Laws in Continuum Physics, Springer-Verlag, Berlin 1999.

[17] R. DiPerna, Global existence of solutions to nonlinear hyperbolic systems of conservation laws, J. Diff. Equat. 20 (1976), 187-212.

[18] R. DiPerna, Convergence of approximate solutions to conservation laws, Arch. Rational Mech. Anal. 82 (1983), 27-70.

[19] J. Glimm, Solutions in the large for nonlinear hyperbolic systems of equations, Comm. Pure Appl. Math. 18 (1965), 697-715.

[20] J. Glimm and P. Lax, Decay of solutions of systems of nonlinear hyperbolic conservation laws, Amer. Math. Soc. Memoir 101 (1970).

[21] J. Goodman and Z. Xin, Viscous limits for piecewise smooth solutions to systems of conservation laws, Arch. Rational Mech. Anal. 121 (1992), 235265. 
[22] H. Holden and N. H. Risebro, Front Tracking for Hyperbolic Conservation Laws, Springer Verlag, New York 2002.

[23] S. Kruzhkov, First order quasilinear equations with several space variables, Math. USSR Sbornik 10 (1970), 217-243.

[24] H. K. Jenssen, Blowup for systems of conservation laws, SIAM J. Math. Anal. 31 (2000), 894-908.

[25] P. Lax, Hyperbolic systems of conservation laws II, Comm. Pure Appl. Math. 10 (1957), 537-566.

[26] T. P. Liu, The entropy condition and the admissibility of shocks, J. Math. Anal. Appl. 53 (1976), 78-88.

[27] T. P. Liu, Decay to N-waves of solutions of general systems of nonlinear hyperbolic conservation laws, Comm. Pure Appl. Math. 30 (1977), 585-610.

[28] T. P. Liu, Linear and nonlinear large-time behavior of solutions of general systems of hyperbolic conservation laws, Comm. Pure Appl. Math. 30 (1977), 767-796.

[29] T. P. Liu, The deterministic version of the Glimm scheme, Comm. Math. Phys. 57 (1977), 135-148.

[30] T. P. Liu, Admissible solutions of hyperbolic conservation laws, Amer. Math. Soc. Memoir 240 (1981).

[31] T. P. Liu and T. Yang, $L^{1}$ stability for $2 \times 2$ systems of hyperbolic conservation laws, J. Amer. Math. Soc. 12 (1999), 729-774.

[32] A. Majda and R. Pego, Stable viscosity matrices for systems of conservation laws, J. Differential Equations 56 (1985), 229-262.

[33] R. Natalini, Recent results on hyperbolic relaxation problems, in Analysis of Systems of Conservation Laws, H. Freisthüler Ed., Chapman \& Hall/CRC, 1998, pp.128-198.

[34] O. Oleinik, Discontinuous solutions of nonlinear differential equations (1957), Amer. Math. Soc. Translations 26, 95-172.

[35] N. H. Risebro, A front-tracking alternative to the random choice method, Proc. Amer. Math. Soc. 117 (1993), 1125-1139.

[36] F. Rousset, Viscous approximation of strong shocks of systems of conservation laws, SIAM J. Math.Anal., to appear.

[37] S. Schochet, Sufficient conditions for local existence via Glimm's scheme for large BV data, J. Differential Equations 89 (1991), 317-354. 
[38] D. Serre, Systems of Conservation Laws I, II, Cambridge University Press, 2000.

[39] J. Smoller, Shock Waves and Reaction-Diffusion Equations, SpringerVerlag, New York, 1983.

[40] A. Vanderbauwhede, Centre manifolds, normal forms and elementary bifurcations, Dynamics Reported, Vol. 2 (1989), 89-169.

[41] S. H. Yu, Zero-dissipation limit of solutions with shocks for systems of hyperbolic conservation laws, Arch. Rational Mech. Anal. 146 (1999), 275370 .

S.I.S.S.A., TRIESTE 34014 , ITALy. 